\title{
¿Statistical Cluster Analysis of Global Aerosol Optical Depth for Simplified Atmospheric Modeling $\mathscr{O}$
}

\author{
NoAh T. Plymale, ${ }^{\mathrm{a}}$ Joshua E. Szekely, ${ }^{\mathrm{a}}$ AND AnNA H. RubinStein ${ }^{\mathrm{a}}$ \\ a System Evaluation Division, Institute for Defense Analyses, Alexandria, Virginia
}

(Manuscript received 25 July 2021, in final form 25 October 2021)

\begin{abstract}
Atmospheric aerosols originating from natural and anthropogenic sources have important implications for modeling atmospheric phenomena, but aerosol conditions can change significantly and rapidly because of their dependence on local geography and atmospheric conditions. In this work, we applied a computational $k$-means clustering algorithm to a global set of data obtained from the Modern-Era Retrospective Analysis for Research and Applications, version 2 (MERRA-2), to yield a set of 25 clusters that discriminate on the basis of land type, elevation, and atmospheric conditions to predict statistical aerosol optical depth (AOD) information. We considered different subsets of MERRA-2 data, consisting of all the data averaged over a single year (2016) as well as data averaged by meteorological season over a span of five years (2012-16), arriving at five separate sets of 25 clusters. We make the clustered AOD information available with decision trees, qualitative cluster descriptions, and color-coded cluster maps to assist in identifying which cluster to use in retrieving AOD information. The results of this analysis have applications in atmospheric modeling where knowledge of approximate or typical aerosol conditions is needed in lookup-table form without requiring access to large atmospheric databases or computationally intensive aerosol models; such applications could include quick-turnaround or large-volume analyses of atmospheric conditions required to inform decision-making that affects national security, such as in modeling remote sensing and estimating upper and lower bounds for visible and infrared photon transport.
\end{abstract}

KEYWORDS: Aerosols; Optical properties; Aerosol optical properties; Clustering; Decision trees; Machine learning

\section{Introduction}

Atmospheric aerosols, which are suspensions of solid or liquid particles in air, participate in Earth's surface energy budget by scattering or absorbing radiation as a function of their size and composition (Rizza et al. 2019; Lohmann and Feichter 2005). Additionally, aerosols can promote or inhibit cloud formation and can contribute to the mechanisms that result in poor breathable air quality (Sun and Ariya 2006; Prospero 1999; Levin and Cotton 2008). The properties of aerosols make them important to consider in developing models for atmospheric phenomena, such as weather models that can predict hazardous conditions (Xian et al. 2019) or atmospheric transmission models important to applications in remote sensing (Berk et al. 1998; Moosmüller et al. 2009).

Aerosol concentrations and compositions are influenced by proximity to geographic features (such as deserts or oceans), atmospheric conditions (including amount of precipitation and wind speed), natural events (such as volcanic activity and forest fires), and human activity (including burning fossil fuels) (Viana et al. 2014). Some of the factors that influence aerosol concentrations can have seasonal dependence, including increased

DDenotes content that is immediately available upon publication as open access.

Supplemental information related to this paper is available at the Journals Online website: https://doi.org/10.1175/JAMC-D-210150.s1.

Corresponding author: Noah T. Plymale, nplymale@ida.org fossil fuel emissions in the winter and increased occurrence of forest fires in the summer. Other factors, such as volcanic activity, can have influence on annual aerosol conditions. The variety and complexity of factors influencing aerosols cause their concentrations in the atmosphere to vary significantly and, often, rapidly, along with their contributions to the atmospheric phenomena they influence (Satheesh et al. 2017). Atmospheric models that require knowledge of aerosol conditions are challenged by the difficulty in predicting aerosol concentrations accurately and quickly.

The Modern-Era Retrospective Analysis for Research and Applications, version 2 (MERRA-2), is a reanalysis of atmospheric conditions provided by the National Aeronautics and Space Administration (NASA) Global Modeling and Assimilation Office encompassing from 1 January 1980 to the present (Gelaro et al. 2017). MERRA-2 includes a substantial database of atmospheric and weather information, including aerosol optical depth (AOD) data, on a grid with $0.5^{\circ}$ latitude $\times$ $0.625^{\circ}$ longitude resolution (Bosilovich et al. 2016). MERRA-2 uses the Goddard Chemistry Aerosol Radiation and Transport model (GOCART; Chin et al. 2002; Colarco et al. 2010), which accounts for organic carbon, black carbon, dust, sea salt, and sulfate aerosols, to describe atmospheric aerosol conditions by providing AOD data for each of these five categories in addition to a total AOD that sums these components. AOD data included in the MERRA-2 model are assimilated from satellite-based sensors, including the Moderate Resolution Imaging Spectroradiometer (MODIS), Multiangle Imaging SpectroRadiometer (MISR), and Advanced Very High Resolution Radiometer (AVHRR), and from the ground-based Aerosol Robotic Network (AERONET) (Holben et al. 1998; Randles et al. 2017; Buchard et al. 2017). 
The total AOD, which quantifies the optical properties for the total aerosols present in the atmosphere, is defined as the natural logarithm of the ratio of incident to transmitted light through a vertical column of the atmosphere excluding contributions from absorption and Rayleigh scattering by air molecules and clouds (Wenny et al. 1998). The AOD is wavelength dependent but is provided by MERRA-2 at $550 \mathrm{~nm}$, which corresponds to the wavelength where aerosol extinction is at a maximum. All references to AOD in this work are specific to the $\mathrm{AOD}$ at $550 \mathrm{~nm}$ (abbreviated $\mathrm{AOD}_{550}$ ) unless otherwise specified. The AOD is a common input that describes aerosol conditions in atmospheric transmission models, including the moderate resolution atmospheric transmission (MODTRAN) model (Berk et al. 2011), which is developed and maintained by Spectral Sciences, Inc., and the Air Force Research Laboratory. MODTRAN uses AOD inputs to compute custom transmission spectra for atmospheric cross sections, and these inputs commonly come from sources such as AERONET, MERRA-2, or deterministic weather prediction models [e.g., the Weather Research and Forecasting Model coupled with Chemistry (WRF-Chem)] that can calculate AOD information as a component of short-term forecasts (Nabavi et al. 2018). MODTRAN is an important transmission model that is widely used in applications pertinent to national security, including in modeling remote sensing and identifying transmission spectra for visible and infrared radiation transport.

We describe herein a process of clustering global geographic and atmospheric data from MERRA-2 using a computational machine-learning technique to find geographic regions having distinct atmospheric conditions, each with accessible statistical AOD information in lookup table form. In clustering the global data, we considered regions of the ocean separate from all other land types, which consist of lake, land, and ice-covered land. We consider annual and seasonal averages of the input dataset and provide 3 "ocean" clusters and 22 "land" clusters for a total of 25 global clusters. The results are presented in a series of tables that contain the cluster information and allow a user to identify an appropriate cluster and its associated AOD information. The result of the analysis is a detailed set of AOD lookup tables organized into clusters that do not require access to a database or computer model, providing data portability and accessibility. We envision that such AOD lookup tables would be useful in performing "what if?" analyses to inform decision-making, particularly for applications in national security and informing operations in defense. Moreover, we believe these lookup tables could provide useful information where access to numerical weather prediction or databases like MERRA- 2 is unavailable or limited, which can be the case on certain networks or potentially in developing countries.

\section{Methods}

The $k$-means algorithm is an unsupervised computational machine-learning technique that groups complex datasets into a defined number of clusters $k$ that have similarity across the input observation vectors (MacQueen 1967). The clusters consist of a set of centroids that closely approximate the mean value for the observations associated with a cluster. The $k$-means algorithm operates by accepting a set of $N$ observation vectors and the desired number of clusters $k$ and returns $k$ clusters that each have $N$ centroids. The algorithm begins with an initialized set of random centroids and iteratively adjusts the centroid positions to minimize the distortion, which is defined as the sum of the squared distance between each observation and its nearest centroid.

The result from the $k$-means algorithm is a code book that maps clusters to their respective centroid values and can be used to assign each set of original observations to the most appropriate cluster. The code book that is ultimately generated is sensitive to the random positions of the initial cluster centroids; local minima in the distortion can result in different code books each time the $k$-means algorithm is executed on the same set of observations. Moreover, the $k$-means algorithm accepts $k$ as an input parameter, requiring the user to identify an optimal value for $k$ prior to beginning. While numerous quantitative methods exist for optimizing $k$, there are situations in which individual methods do not yield clear, conclusive, or consistent answers; the methods are sensitive to the nature of the dataset and the results are best interpreted with respect to each other and in the context of the problem at hand.

An ideal value for $k$ maximizes the information represented by the clusters while conserving computational resources required for clustering. Appendix A details the methodology and logic we used in optimizing the number of clusters for the MERRA-2 input variables (Satopää et al. 2011; Tolsa 2000; Zhao et al. 2008). We compared four methods for optimizing $k$ and considered values only where multiple methods were in agreement. Additionally, we restricted the range of $k$ that we considered so as to group so-called ocean (fraction of ocean $=$ 1) and so-called land (i.e., not exclusively ocean, or fraction of ocean $<1)$ data subsets into clusters according to the projected relative interest in identifying an $\mathrm{AOD}_{550}$ for locations in these data subsets. We assumed lower specific interest for the quick-turn or large-volume applications relevant to national security envisioned for these lookup tables in modeling the $\mathrm{AOD}_{550}$ over the open ocean and consequently limited the number of ocean clusters, while accommodating a larger number of clusters over regions containing land, ice-covered land, or lake. Further, we empirically determined that the clusters followed geography more sensibly and could better serve our envisioned applications in supporting decision-making when the ocean and land datasets were clustered separately. As mentioned in the introduction, we ultimately divided ocean regions into 3 clusters and land regions into 22 clusters for a total of 25 global clusters.

The $k$-means algorithm is well suited to finding correlations between atmospheric aerosols and natural factors that influence them, such as geography and ambient weather, because its distortion minimization algorithm is intended to identify correlations among sets of observations (Govender and Sivakumar 2019; Liu and George 2005; Wegner et al. 2012). We accounted for geography using land type and elevation variables that we derived from the MERRA-2 database. Further, we accounted for ambient weather in each MERRA-2 grid cell by including relative humidity, 
temperature, wind speed, and fractions of showers (the fraction of the MERRA-2 grid cell experiencing showers) and cloud cover as inputs to the clustering algorithm. We also indirectly include aerosol contributions from natural and anthropogenic sources by including the AOD variables for the five aerosol components and the total AOD in the cluster analysis. Detailed information on the variables derived from MERRA-2 is provided in section 3 .

We extracted MERRA-2 data for the years 2012-16 and averaged the data either annually or according to the meteorological season desired. We selected relatively recent years for this analysis to account for recent trends in global aerosol conditions better than would be possible by randomly sampling years available in MERRA-2. Computational and resource limitations precluded analysis of all available MERRA-2 reanalysis data. Additionally, by averaging the AOD data from MERRA-2, which is a necessary step in performing the clustering process, we reduce sensitivity of the clustered results to short-lived aerosol events (e.g., fires) and rapid changes in wind direction, which can influence aerosol sources in some geographic regions. As aerosol conditions continue to change with natural events and human activities, we expect this technique may require continuous analysis of recent MERRA-2 results, but ideally such analysis would be performed after further refinement of the methods and engagement with the community.

We stored the MERRA-2 data as linear observation vectors and created separate datasets to represent ocean (fraction of ocean $=1$ ) and land (fraction of ocean $<1$ ) subsets of the data. We used the "whiten" function available in the SciPy package (version 1.3.2) implemented in Python (version 3.7) to give the data unit variance by dividing each data point by the standard deviation of the data in the corresponding vector. We then used the "kmeans" function of the SciPy package to generate a code book containing the cluster centroids using $k=3$ clusters for the ocean subset and using $k=22$ clusters for the land subset of the data. Using SciPy's "vq" function, we mapped each set of observations to a centroid using the appropriate code book, removed the whiten processing on the code book by multiplying each entry by the standard deviation of the data in each observation vector, and made the results available as a tool accessible in the online supplemental material provided with this work.

\section{Data sources}

Table 1 summarizes the variables considered in the $k$ means clustering analysis and gives the corresponding MERRA-2 name(s) (Bosilovich et al. 2016). Variables that were calculated from raw MERRA-2 data are the elevation, relative humidity, 10-m wind speed, fraction of showers, fraction of black carbon, fraction of dust, fraction of organic carbon, fraction of sea salt, and fraction of sulfate. The fractional contribution to total AOD for each of the five aerosol types was computed by finding the ratio of each aerosol component AOD to the total AOD $_{550}$ (TOTEXTTAU in MERRA-2).
The elevation $h$ was calculated from the surface geopotential height ( $\mathrm{SGH} ; \mathrm{m}^{2} \mathrm{~s}^{-2}$ ) provided in MERRA-2 using (Leroy 1997)

$$
h=\frac{\left(6.356 \times 10^{6}\right) \mathrm{SGH} / 9.8}{6.356 \times 10^{6}-(\mathrm{SGH} / 9.8)}
$$

Here, we approximate Earth as a sphere with a radius of $6.356 \times 10^{6} \mathrm{~m}$ and assume the acceleration due to gravity is constant at $9.8 \mathrm{~m} \mathrm{~s}^{-2}$.

The relative humidity was calculated from the surface pressure $p$, the 2-m specific humidity $q$, and 2-m air temperature $T$ using the following method. First, the measured mass mixing ratio $w$ of water vapor to dry air was calculated (Wallace and Hobbs 2006) as

$$
w=\frac{q}{1-q} .
$$

Then, the saturation vapor pressure $e_{s}$ was calculated above liquid water (where $T \geq 0^{\circ} \mathrm{C}$ ) and above ice (where $T<0^{\circ} \mathrm{C}$ ) respectively using A. Buck's two equations (Buck 1981):

$$
\begin{aligned}
& e_{s, T \geq 0{ }^{\circ} \mathrm{C}}=611.21 \exp \left[\left(18.678-\frac{T}{234.5}\right)\left(\frac{T}{257.14+T}\right)\right] \text { and } \\
& e_{s, T<0{ }^{\circ} \mathrm{C}}=611.15 \exp \left[\left(23.036-\frac{T}{333.7}\right)\left(\frac{T}{279.82+T}\right)\right] .
\end{aligned}
$$

Here, $T$ is in degrees Celsius. The saturation vapor pressure was converted to the saturation mass mixing ratio of water to dry air at equilibrium $w_{s}$ (Wallace and Hobbs 2006) assuming that the surface pressure $p$ is approximated by the 2-m surface pressure variable in MERRA-2:

$$
w_{s}=0.622 \frac{e_{s}}{p-e_{s}} \text {. }
$$

The relative humidity $(\mathrm{RH})$ was then calculated (Wallace and Hobbs 2006):

$$
\mathrm{RH}=\frac{w}{w_{s}} \times 100 \% .
$$

The 10-m wind speed $W_{T}$ was calculated from the $10-\mathrm{m}$ eastward and northward wind speeds $\left(W_{E}\right.$ and $W_{N}$, respectively) using

$$
W_{T}=\sqrt{W_{E}^{2}+W_{N}^{2}}
$$

The areal fraction of showers $S_{F}$ was calculated for each MERRA-2 grid cell by finding the maximum of the variables representing the fraction of anvil showers $S_{A}$, the fraction of convective showers $S_{C}$, and the fraction of nonanvil largescale showers $S_{\mathrm{LS}}$. The method used to find $S_{F}$ is 
TABLE 1. MERRA-2 variables used for $k$-means clustering.

\begin{tabular}{|c|c|c|c|}
\hline Variable name & Units & Description & MERRA-2 name \\
\hline Fraction of lake & - & $\begin{array}{l}\text { Fraction of the MERRA-2 } \\
\text { grid cell that is categorized } \\
\text { as lake }\end{array}$ & FRLAKE \\
\hline Fraction of land & - & $\begin{array}{l}\text { Fraction of the MERRA-2 } \\
\text { grid cell that is categorized } \\
\text { as land }\end{array}$ & FRLAND \\
\hline Fraction of land ice & - & $\begin{array}{l}\text { Fraction of the MERRA-2 } \\
\text { grid cell that is categorized } \\
\text { as ice-covered } \\
\text { land-commonly } \\
\text { permafrost regions }\end{array}$ & FRLANDICE \\
\hline Fraction of ocean & - & $\begin{array}{l}\text { Fraction of the MERRA-2 } \\
\text { grid cell that is categorized } \\
\text { as ocean }\end{array}$ & FROCEAN \\
\hline Elevation & $\mathrm{m}$ & $\begin{array}{l}\text { Elevation is derived from the } \\
\text { surface geopotential height } \\
\left(\mathrm{m}^{2} \mathrm{~s}^{-2}\right) \text { by assuming } \\
\text { Earth's radius as } 6356 \mathrm{~km} \\
\text { and the acceleration due to } \\
\text { gravity as } 9.8 \mathrm{~m} \mathrm{~s}^{-2}\end{array}$ & PHIS \\
\hline RH & $\%$ & $\begin{array}{l}\text { Relative humidity is derived } \\
\text { from the surface pressure } \\
(\mathrm{Pa}), 2-\mathrm{m} \text { specific humidity, } \\
\text { and 2-m air temperature } \\
(\mathrm{K})\end{array}$ & PS, QV2M, T2M \\
\hline 2-m air temperature & $\mathrm{K}$ & $\begin{array}{l}\text { Air temperature } 2 \mathrm{~m} \text { above } \\
\text { the surface }\end{array}$ & $\mathrm{T} 2 \mathrm{M}$ \\
\hline 10-m wind speed & $\mathrm{m} \mathrm{s}^{-1}$ & $\begin{array}{l}\text { Wind speed } 10 \mathrm{~m} \text { above the } \\
\text { surface, derived from the } \\
\text { 10-m eastward and northward } \\
\text { wind speeds }\left(\mathrm{m} \mathrm{s}^{-1}\right)\end{array}$ & U10M, V10M \\
\hline Fraction showers & - & $\begin{array}{l}\text { The areal fraction of showers } \\
\text { is defined as the maximum } \\
\text { value across the variables } \\
\text { representing the areal } \\
\text { fraction of anvil showers, } \\
\text { convective showers, and } \\
\text { nonanvil large-scale } \\
\text { showers }\end{array}$ & FRCAN, FRCCN, FRCLS \\
\hline Fraction cloud coverage & - & Areal fraction of clouds & CLDTOT \\
\hline Fraction black carbon & - & $\begin{array}{l}\text { Fractional contribution of } \\
\text { black carbon aerosols to } \\
\text { total AOD at } 550 \mathrm{~nm}\end{array}$ & BCEXTTAU \\
\hline Fraction dust & - & $\begin{array}{l}\text { Fractional contribution of dust } \\
\text { aerosols to total AOD at } \\
550 \mathrm{~nm}\end{array}$ & DUEXTTAU \\
\hline Fraction organic carbon & - & $\begin{array}{l}\text { Fractional contribution of } \\
\text { organic carbon aerosols to } \\
\text { total AOD at } 550 \mathrm{~nm}\end{array}$ & OCEXTTAU \\
\hline Fraction sea salt & - & $\begin{array}{l}\text { Fractional contribution of sea- } \\
\text { salt aerosols to total AOD } \\
\text { at } 550 \mathrm{~nm}\end{array}$ & SSEXTTAU \\
\hline Fraction sulfate & - & $\begin{array}{l}\text { Fractional contribution of } \\
\text { sulfate aerosols to total } \\
\text { AOD at } 550 \mathrm{~nm}\end{array}$ & SUEXTTAU \\
\hline Total AOD & - & $\begin{array}{l}\text { Total aerosol optical depth at } \\
550 \mathrm{~nm}\end{array}$ & TOTEXTTAU \\
\hline
\end{tabular}




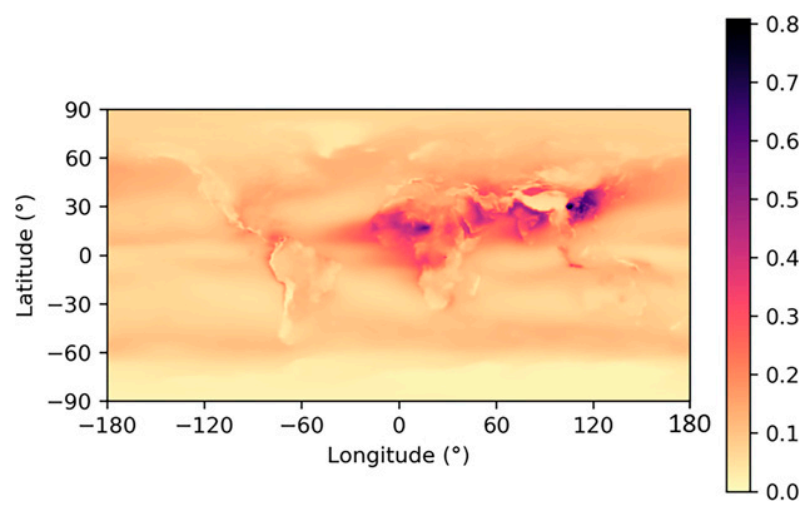

FIG. 1. Fiftieth-percentile $\mathrm{AOD}_{550}$ for the full 2012-16 global MERRA-2 dataset.

$$
S_{F}=\max \left(S_{A}, S_{C}, S_{\mathrm{LS}}\right)
$$

In this analysis, we considered MERRA-2 data for five periods of time: 1) all of the data available for 2016 in addition to the four meteorological seasons for the years 2012-16, which are 2) December-February, 3) March-May, 4) JuneAugust, and 5) September-November. The data corresponding to these time periods were extracted from the MERRA-2 database and averaged over all days in the applicable date range before clustering the data. To visualize the data that we extracted from MERRA-2, Fig. 1 depicts the 50th percentile of the global total AOD $_{550}$ for 2012-16.

To compare our reduced $\mathrm{AOD}_{550}$ dataset identified by the $k$-means algorithm with experimental data, we used data from the AERONET system of sun photometers (Giles et al. 2019). Since the AOD $_{550}$ data available in MERRA-2, and thereby included in the cluster analysis, are partially informed by this AERONET data, we consider this comparison a limited-scope verification and validation. While this analysis does not include a comparison with a completely independent set of experimental AOD data, it does inform a level of expectation for using the clustering results to identify an $\mathrm{AOD}_{550}$ value to use for an application. We extracted data from 30 AERONET sites to compare with the statistical $\mathrm{AOD}_{550}$ found for the clusters. The AERONET sites we analyzed had AOD data collected daily at 555, 551, and/or $532 \mathrm{~nm}$, which were the three closest standard wavelengths to $550 \mathrm{~nm}$ for which AOD data were available from AERONET. For simplicity, we did not attempt to scale the AERONET AOD to $550 \mathrm{~nm}$ and instead assumed that the difference in AOD for wavelengths in the range $532-555 \mathrm{~nm}$ is negligible. We used daily average level-2.0 AERONET, version 2, data (accessed 21 October 2017; https://aeronet.gsfc.nasa.gov/new_web/aerosols.html)—which are quality assured, cloud cleared, and manually inspected - in this comparison.

\section{Results}

The result of performing the $k$-means algorithm on the MERRA-2 variables summarized in Table 1 is a code book

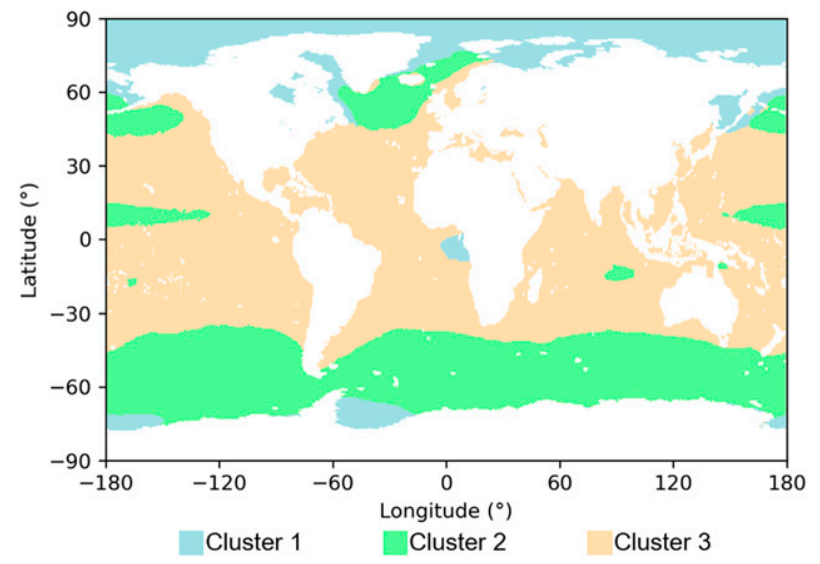

FIG. 2. Geographic location of the clusters identified from the ocean subset of the full 2016 global MERRA-2 dataset. The clusters are ordered by average temperature, where the coolest region is cluster 1 and the warmest region is cluster 3 .

that contains the centroids for each of the clusters identified by the algorithm. The code book is then used to retroactively assign the observations at each location in the original global dataset to the cluster that best represents the data. The result is an array of integers that assigns each of the global MERRA-2 grid cells to a cluster.

Figure 2 shows the geographic location of the three clusters belonging to the ocean subset of the processed annual 2016 MERRA-2 data. In the geographic cluster plots, the clusters are ordered by their centroid temperature, where light blue represents cooler temperatures and light orange represents warmer temperatures. In the clustered ocean data, cool regions of the ocean near the poles with low wind speed and low total AOD (due to low sea-salt aerosol contribution) tended to cluster together, with the exception of an area off the western coast of Africa, where annual biomass burning increases the organic carbon aerosol contribution. The lightgreen cluster in Fig. 2, which has an average daily temperature between the light-blue and light-orange clusters and the highest wind speed, is discontinuous and contains cool regions in the midlatitudes, particularly the Southern Ocean, and equatorial regions of the Pacific and Indian Oceans, with total AOD that is dominated by contributions from sea-salt aerosols. The discontinuity in the light-green and, to a lesser extent, light-blue cluster regions is a result of the $k$-means algorithm clustering regions of the ocean where variables other than the temperature show correlation, including regions with similar humidity, wind speed, precipitation, cloud cover, and component AOD contributions. The small number of clusters included over the open ocean is not able to capture the full extent of climate and aerosol variability that occurs over the ocean, but the results show three characteristic regions with distinct properties.

Figure 3 shows four panels depicting the 22 land clusters (numbered clusters 4-25) identified by the $k$-means algorithm for the global 2016 averaged MERRA-2 dataset. These clusters are also ordered by their temperature centroid, where 

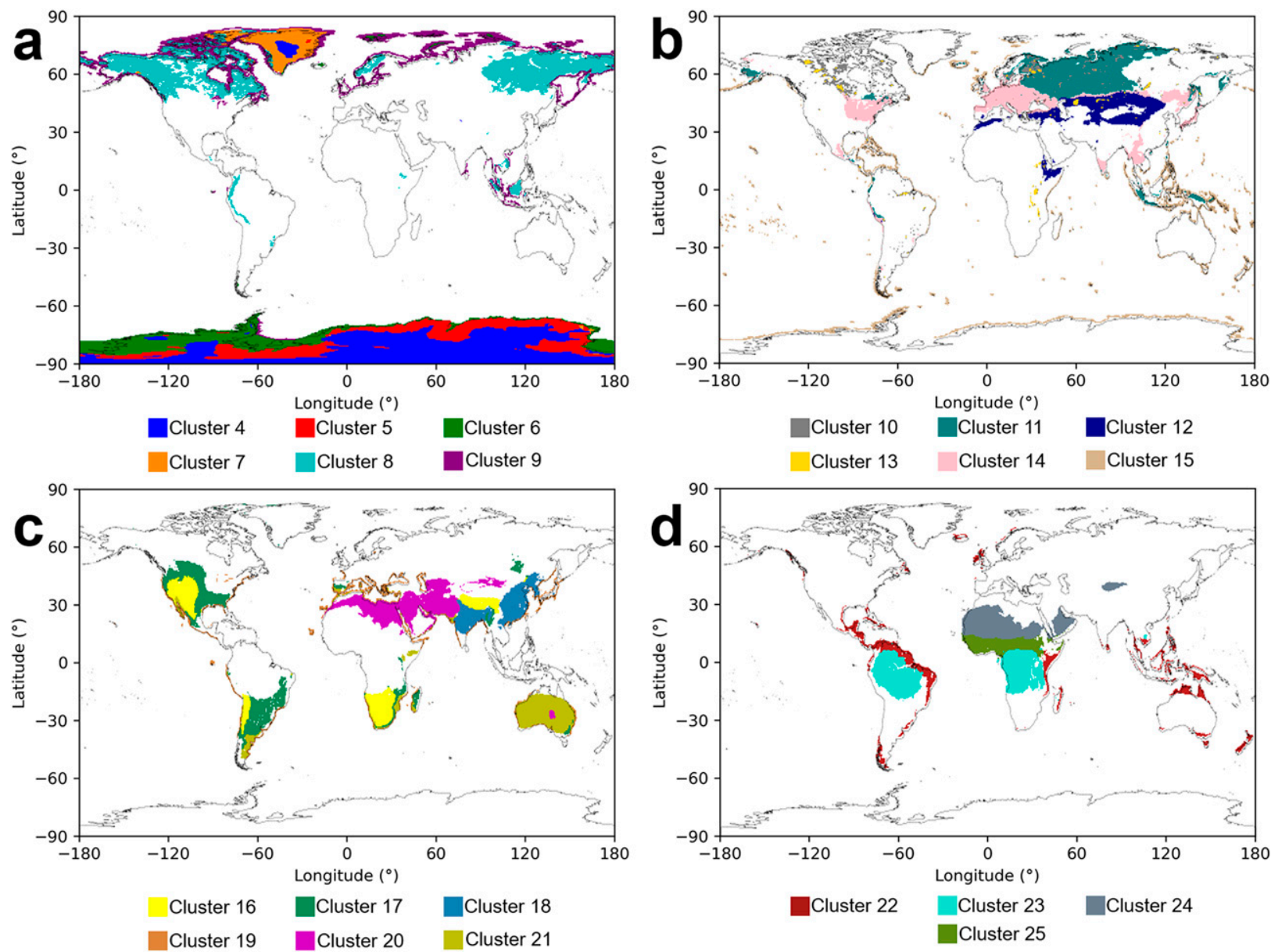

FIG. 3. Geographic location of the clusters identified from the land subset of the full 2016 global MERRA-2 dataset. The cluster numbers are ordered by temperature centroid, where the temperature increases with cluster number. The six coldest clusters are primarily located in Antarctica, Greenland, Russia, and Canada.

increasing cluster number corresponds with increasing temperature centroid. The $k$-means algorithm was able to cluster a number of regions around the globe that intuitively belong to the same group and have similar aerosol sources, a key driver in predicting AOD. For example, the tropical rain forest regions of equatorial South America and Africa are grouped together in cluster 23, which has warm temperatures, high cloud coverage, high contributions from organic carbon aerosols (biomass burning), and very low wind speed. The algorithm also identified cluster 18 as consisting of eastern China, the Korean Peninsula, and much of India that coincides with locations having high $\mathrm{AOD}_{550}$, mostly driven by sulfate aerosols, indicated by dark regions in Fig. 1. In addition, the majority of the Sahara and the southern portion of the Arabian Peninsula compose cluster 24, which had total AOD $_{550}$ dominated by contributions from dust aerosols. Although not always clearly depicted in Fig. 3, the algorithm also separated coastal regions, areas with inland lakes, and regions of permafrost from inland areas without these features. Clusters with these geographic features are best visualized in the individual cluster maps available in the online supplemental material.
The five sections in the online supplemental material each contain the code books and global cluster assignments for MERRA-2 data averaged over the specified periods of time. Table 2 details which online supplemental section corresponds to which time period of MERRA-2 data that was considered in the clustering process. Each of these supplemental sections presents the results in the same format. The code books contain the centroid data for each cluster, but identifying a cluster of interest directly from a code book is onerous because of the level of specificity contained in the cluster centroids.

TABLE 2. Time of year corresponding to the data in each section of the online supplemental material (SM).

\begin{tabular}{cl}
\hline \hline SM section & Time period considered \\
\hline S-A & All of 2016 \\
S-B & Dec/Jan/Feb (2012-16) \\
S-C & Mar/Apr/May (2012-16) \\
S-D & Jun/Jul/Aug (2012-16) \\
S-E & Sep/Oct/Nov (2012-16) \\
\hline
\end{tabular}


To assist users in identifying an appropriate $\mathrm{AOD}_{550}$ to use for atmospheric transmission modeling in support of quickturn analysis and decision-making, we have provided several tools that help identify a suitable cluster. First, each of the MERRA-2 cluster code books has a unique decision tree that assists users in narrowing the set of clusters under consideration according to specific criteria, such as land type and elevation. Second, we provide a qualitative description that categorizes the most intuitive cluster variables and describes associated characteristic regions for each cluster. Last, we provide global color-coded maps for each of the individual clusters to assist in visual identification of the region of interest. The remainder of this section describes the contents of and intended use for each of these tools by providing a walkthrough example of the results for the ocean clusters from the 2016 annual average MERRA-2 data in the main text and describing the full results available in the online supplemental material.

Table 3 presents an excerpt of the code-book clustering results summarizing the centroids for the three ocean clusters identified from the global 2016 MERRA-2 dataset. The first four variables (fraction of lake, land, land ice, and ocean) categorize the clusters according to land type (which is ocean for the clusters in Table 3), and the last column provides the centroids found for the total $\mathrm{AOD}_{550}$. The full code book corresponding to each set of MERRA-2 data that we analyzed is provided in the first table of each section of the online supplemental material (e.g., Table S-A1). The cluster numbers are color coded with the predominant land type for each cluster, where the first three clusters always correspond to the ocean subset of the data.

In all cases, the centroid for fraction of showers is greater than the centroid for fraction of cloud coverage. This result is not intuitive, assuming cloud coverage is required to produce showers. We observed this phenomenon in the raw MERRA2 dataset for individual grid cells as well. Consequently, we recommend that these two variables be considered relative to the dataset as a whole and encourage use of the cluster qualitative descriptors to understand these variables and relate them to measurements of precipitation and cloud cover.

The second and third tables in each of the sections in the online supplemental material (e.g., Tables S-A2 and S-A3) provide the 10th, 25th, 50th, 75th, and 90th percentiles of the global ocean and land data, respectively, for each of the clustered variables. We used these percentiles to assign qualitative descriptors that categorize the centroids of the following five variables: humidity, temperature, wind speed, fraction of showers, and fraction of cloud coverage. We selected these five variables because they provide an intuitive understanding of typical atmospheric conditions for a given cluster, allowing users to get a sense of the characteristic climate within a cluster. Our intended applications for the clustering results is in atmospheric transmission modeling, which considers the whole atmosphere, of which aerosols are only one part, so it makes sense to consider broad atmospheric conditions in our categorization. The qualitative descriptors resulting from these percentile designations effectively bin each of the cluster centroids for these categories into a percentile range to assist in describing the clusters 
TABLE 4. Cluster qualitative descriptor definitions.

\begin{tabular}{cc}
\hline \hline Cluster centroid descriptor & Percentile range \\
\hline Very low & $0-10$ \\
Low & $10-25$ \\
Medium low & $25-50$ \\
Medium high & $50-75$ \\
High & $75-90$ \\
Very high & $90-100$ \\
\hline
\end{tabular}

relative to each other. Qualitative descriptions of these variables further provide a means of identifying a cluster with a set of desired properties. Table 4 maps each qualitative descriptor to a percentile range.
The descriptors defined in Table 4 are provided in Table 5 for the five relevant variables for each of the three ocean clusters from the annual 2016 average MERRA-2 dataset, along with characteristic regions and $\mathrm{AOD}_{550}$ statistics for each cluster. For clusters over land, we also characterize the predominant land type and the elevation to better describe the cluster geography, which can influence aerosol sources. The AOD centroid and AOD average are typically very similar values; however, the centroid is calculated through an iterative process by the $k$-means algorithm and sometimes deviates slightly from the arithmetic average. We provide the standard deviation to give a sense of the spread of the $\mathrm{AOD}_{550}$ within a cluster. We additionally provide the 10th-, 50th-, and 90thpercentile $\mathrm{AOD}_{550}$ to represent low aerosol (high visibility),

TABLE 5. Cluster descriptions and $\mathrm{AOD}_{550}$ statistics for the ocean clusters of the full 2016 global MERRA-2 dataset extracted from Table S-A4 of the online supplemental material.

\begin{tabular}{|c|c|c|c|c|c|c|c|c|}
\hline Cluster & $\begin{array}{l}\text { Qualitative } \\
\text { description }\end{array}$ & $\begin{array}{c}\text { Characteristic } \\
\text { regions }\end{array}$ & AOD centroid & AOD avg & AOD std dev & $\begin{array}{l}\text { AOD 10th } \\
\text { percentile }\end{array}$ & $\begin{array}{l}\text { AOD 50th } \\
\text { percentile }\end{array}$ & $\begin{array}{l}\text { AOD 90th } \\
\text { percentile }\end{array}$ \\
\hline 1 & $\begin{array}{l}\text { Regions of the } \\
\text { oceans: } \\
\text { humidity is } \\
\text { high, } \\
\text { temperature } \\
\text { is low, wind } \\
\text { speed is } \\
\text { medium low, } \\
\text { showers are } \\
\text { medium high, } \\
\text { and cloud } \\
\text { coverage is } \\
\text { high }\end{array}$ & $\begin{array}{l}\text { Arctic Ocean, } \\
\text { Hudson Bay, } \\
\text { Sea of } \\
\text { Okhotsk, } \\
\text { northern } \\
\text { Bering Sea, } \\
\text { and } \\
\text { southernmost } \\
\text { portion of the } \\
\text { Southern } \\
\text { Ocean }\end{array}$ & 0.0850 & 0.0847 & 0.0461 & 0.0624 & 0.0741 & 0.110 \\
\hline 2 & $\begin{array}{l}\text { Regions of the } \\
\text { oceans: } \\
\text { humidity is } \\
\text { medium high, } \\
\text { temperature } \\
\text { is medium } \\
\text { low, wind } \\
\text { speed is high, } \\
\text { showers are } \\
\text { high, and } \\
\text { cloud } \\
\text { coverage is } \\
\text { medium high }\end{array}$ & $\begin{array}{l}\text { Median } \\
\text { temperature } \\
\text { regions of the } \\
\text { Southern and } \\
\text { Atlantic } \\
\text { Oceans, } \\
\text { including a } \\
\text { central } \\
\text { portion of the } \\
\text { Pacific Ocean }\end{array}$ & 0.102 & 0.102 & 0.0261 & 0.0629 & 0.105 & 0.132 \\
\hline 3 & $\begin{array}{l}\text { Regions of the } \\
\text { oceans: } \\
\text { humidity is } \\
\text { medium low, } \\
\text { temperature } \\
\text { is medium } \\
\text { high, wind } \\
\text { speed is } \\
\text { medium low, } \\
\text { showers are } \\
\text { low, and } \\
\text { cloud } \\
\text { coverage is } \\
\text { low }\end{array}$ & $\begin{array}{l}\text { Majority of } \\
\text { oceans and } \\
\text { seas in } \\
\text { midlatitudes; } \\
\text { extends in an } \\
\text { arc past the } \\
\text { British Isles } \\
\text { and north of } \\
\text { Norway }\end{array}$ & 0.120 & 0.120 & 0.0620 & 0.0700 & 0.103 & 0.192 \\
\hline
\end{tabular}




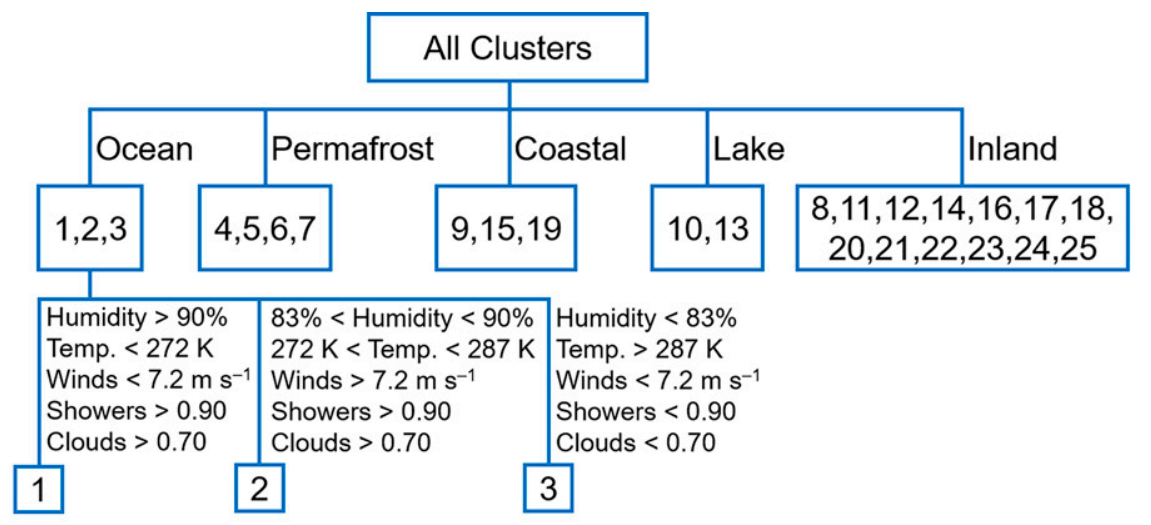

FIG. 4. Partial decision tree for clusters describing the full 2016 global MERRA-2 dataset extracted from Fig. S-A1 in the online supplemental material.

typical aerosol (typical visibility), and high aerosol (low visibility) conditions, respectively. As the MERRA-2 data were averaged over time, these statistical metrics pertain to the geographic region encompassed by a cluster and are not temporal in nature. The full set of cluster descriptions and characteristic regions are available for each MERRA-2 dataset analyzed in the fourth table of each section of the online supplemental material (e.g., Table S-A4).

Figure 4 depicts a portion of a decision tree for the global 2016 MERRA-2 clustering results to provide an example of how the three ocean clusters break down by humidity, temperature, wind speed, fraction of showers, and fraction of cloud coverage. Figure 4 is an excerpt of the full decision tree (Fig. S-A1, available in the online supplemental material), which breaks apart all 25 of the clusters and has many additional branches that attempt to systematically distinguish clusters based on the relevant centroids of the variables considered. The seven variables considered in the full decision trees are land type (an interpretation of fraction of lake, land, land ice, and ocean), elevation, humidity, temperature, wind speed, fraction of showers, and fraction of cloud coverage. We used quantitative metrics to divide clusters in the decision trees using these variables; we grouped clusters that had similar centroids for a particular variable. We generally arrived at these quantitative delineations by calculating the midpoint between adjacent cluster centroids.

Distinguishing between different clusters using the decision trees is sensitive to the order in which the quantitative delineations are made between clusters (e.g., whether a cluster is separated by its humidity before temperature or vice versa), particularly for similar inland clusters. We recommend using the decision trees sparingly and in conjunction with the other available representations of the cluster data to arrive at the best cluster for modeling an atmosphere that appropriately represents the relevant national security question at hand. All of the full decision trees breaking apart the clusters according to their unique cluster centroids are available as the first figure in each section of the online supplemental material (e.g., Fig. S-A1).

The list of characteristic regions provided for the three ocean clusters in Table 5 is not exhaustive, so Fig. 5 graphically breaks apart the individual cluster maps to better depict the cluster geography for the ocean clusters from Fig. 2. This is particularly helpful for land clusters that represent disconnected or narrow regions, the full extent of which are provided in the second through seventh figures of each section of the online supplemental material (e.g., Figs. S-A2-S-A7). These figures are useful for identifying the geographic extent of a region and can help with developing an intuitive picture of what a region represents on a global scale. Using the graphical representation of the clusters in conjunction with the decision tree and the qualitative description of the cluster centroids provides the user with a comprehensive means of identifying an appropriate cluster to model atmospheric aerosols in supporting defense-related studies modeling visible and infrared radiation transport or remote sensing, for example.

We recommend the following steps for using the online supplemental material to find the most appropriate cluster for the modeling to be performed. We use "S-X" as a stand-in for S-A, S-B, S-C, S-D, and S-E to illustrate to which supplemental figure or table to refer.

1) Use Table 2 to decide which section of the online supplemental material to refer to according to the period appropriate for modeling to be performed.

2) Determine which cluster best represents the conditions and region of interest.

(i) Use the decision tree in Fig. S-X1 to identify which clusters correspond to the land type of interest. The decision tree can be consulted to further narrow the subset of clusters under consideration, but we caution against using the decision tree as the only method for cluster identification.

(ii) Consult Fig. S-X2-S-X7 to identify candidate clusters according to their graphical representations on a map.

(iii) Consult the qualitative description of the candidate clusters in Table S-X4 and use these descriptions to narrow the clusters under consideration by their characteristics.

(iv) If a specific cluster is not yet identified, refer back to the decision tree in Fig. S-X1, the characteristic 

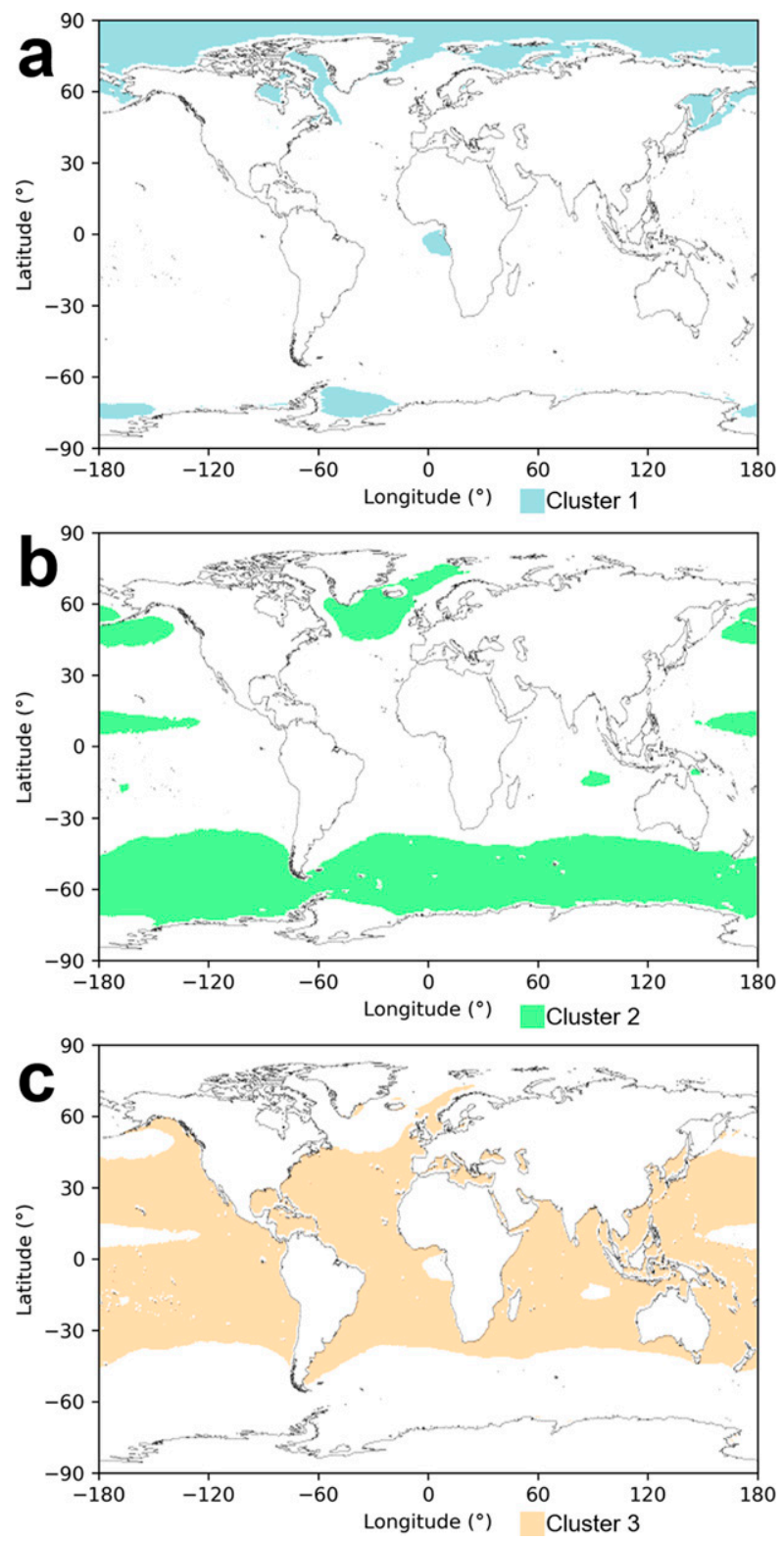

FIG. 5. Geographic extent of the ocean clusters from the full 2016 global MERRA-2 dataset.

regions in Table $\mathrm{S}-\mathrm{X} 4$, and the graphical cluster representations in Fig. S-X2-S-X7 to further narrow the clusters under consideration.

3) Find the cluster number in Table $S-X 4$ and record the last three columns, which are the 10th-, 50th-, and 90th-percentile AOD. These represent low, typical, and high aerosol conditions, respectively, for that cluster.

\section{Discussion}

The AOD is an important parameter in modeling atmospheric phenomena, as evidenced by dedicated studies of aerosol climatology (Kinne et al. 2013). The AOD $_{550}$ data tables and cluster maps in the online supplemental material are intended to provide a fast means of estimating the $\mathrm{AOD}_{550}$ without requiring access to a large database like MERRA-2 or requiring the time to run computer models to make estimates for $\mathrm{AOD}_{550}$. However, the $k$-means algorithm, which is an unsupervised machine-learning technique used for identifying the clusters, does not assess the accuracy of the results and cannot determine whether using the $\mathrm{AOD}_{550}$ statistics given for each cluster is appropriate for a given application.

In a limited assessment of the dependability of the $\mathrm{AOD}_{550}$ data available for each cluster, we compared experimental AOD data from AERONET sites with the statistical AOD 550 data reported in this work for the cluster in which each site is located. This assessment is limited because, of 996 candidate sites, only 30 , whose locations are depicted in appendix B, had AOD data available for any of the desired wavelengths, and many sites were located in the same cluster; a total of 7 unique clusters contained AERONET sites for each time of year. Additionally, AERONET data are assimilated in MERRA-2 AOD 550 data, and our comparison examines how different the clustered $\mathrm{AOD}_{550}$ results are from original AERONET data after assimilation in MERRA-2 followed by $k$-means clustering. Moreover, the comparison does not attempt to assess the accuracy of MERRA-2 AOD predictions directly but instead examines the distance between the predicted cluster $\mathrm{AOD}_{550}$ results, which are inherently based on MERRA-2, and the corresponding AERONET AOD measurements.

We compared the average AERONET AOD at 555, 551, and/or $532 \mathrm{~nm}$ for all days contained within the respective date range with the average $\mathrm{AOD}_{550}$ for the cluster in which each AERONET site is located. We also compared the 10th-, 50th-, and 90th-percentile AOD at each candidate AERONET site with the percentiles for the corresponding cluster. We found the magnitude of the difference (absolute value) between the AOD statistic computed from AERONET data and the respective cluster $\mathrm{AOD}_{550}$ statistic and divided by the AERONET statistic to compute the fractional difference between the actual (AERONET) and predicted (cluster) AOD result. Table 6 presents the average of these fractional differences across all statistical metrics compared. Appendix $\mathrm{B}$ contains plots that show all of the AERONET AOD data versus the corresponding cluster $\mathrm{AOD}_{550}$ data considered in this analysis.

The results of this limited assessment of the accuracy of the clustered AOD data shown in Table 6 and appendix B demonstrate that the statistical $\mathrm{AOD}_{550}$ data provided for the clusters can vary substantially from experimentally measured AERONET AOD data. However, these results are far from comprehensive, as we only compared a small subset of cluster data with AERONET data. Much of the AERONET AOD data were collected during years prior to the 2012-16 years from which the clustered data were extracted, and given that some factors including volcanic activity can influence AOD on an annual basis, inherent differences in the AERONET AOD measurements and the clustered AOD $_{550}$ results could 
TABLE 6. Comparison of AERONET and clustered AOD data.

\begin{tabular}{lccc}
\hline \hline & $\begin{array}{c}\text { Avg fractional error in } \\
\text { clustered AOD data vs } \\
\text { AERONET }\end{array}$ & $\begin{array}{c}\text { Avg spread of AERONET } \\
\text { AOD data }\end{array}$ & $\begin{array}{c}\text { Spread of clustered AOD }^{\text {b50 }} \text { data }^{{ }^{c}} \\
\text { Time of year }\end{array}$ \\
\hline Annual & 0.58 & 0.68 & 0.34 \\
Dec-Feb & 1.0 & 0.63 & 0.34 \\
Mar-May & 0.58 & 0.60 & 0.42 \\
Jun-Aug & 0.49 & 0.61 & 0.35 \\
Sep-Nov & 0.76 & 0.70 & 0.33 \\
\hline
\end{tabular}

${ }^{a}$ The fractional error in clustered AOD data is the average across the three considered AERONET wavelengths of the absolute difference between the AERONET AOD statistic and the respective cluster AOD statistic divided by the AERONET AOD statistic.

b The average spread of AERONET AOD data is the average for all three wavelengths of the standard deviation for the daily AERONET AOD data available for the corresponding time of year divided by the average AOD for that time period.

${ }^{c}$ The spread of the clustered AOD data is the average of the standard deviation of the AOD divided by the average AOD within all clusters.

be present. Moreover, the comparison between AERONET AOD and cluster AOD $_{550}$ predictions does not attempt to select AERONET data collected during atmospheric conditions that correspond with the conditions identified in the clustering process, potentially exacerbating differences between measured and predicted AOD.

To give further context, Table 6 also presents the average spread of the AERONET data, which is a representation of the standard deviation of the AOD as a fraction of the average AOD, for each respective time of year. The spread in the AERONET AOD data can be significant and, with the exception of the December-February data, is greater than the average error observed from the cluster $\mathrm{AOD}_{550}$ prediction. Because the error in the cluster $\mathrm{AOD}_{550}$ prediction falls within the spread of the AERONET AOD data for most date ranges, the expectation is that the $\mathrm{AOD}_{550}$ predicted by the clusters can represent most AOD conditions for situations where simplicity and speed are desired in exchange for fidelity of the AOD estimate; such applications could include performing "what if?" or quick-turnaround analyses that support national security.
The results of the comparison in Table 6 are far from definitive, but they do emphasize that, given the unpredictable nature of atmospheric aerosols, atmospheric models that account for aerosols should consider the statistical spread of the AOD information available. Perhaps the best solution is to anticipate a realistic range of conditions to model that consider extreme and typical conditions together. We included the 10th-, 50th-, and 90th-percentile $\mathrm{AOD}_{550}$ data for each cluster in each section of the online supplemental material to provide a way of accounting for the spread in AOD where lower fidelity AOD statistics are sufficient and for applications that may need to identify an appropriate range of aerosol conditions. In particular, we expect that the lower (10th percentile) and upper (90th percentile) AOD results would assist decision-makers in bounding the aerosol conditions when modeling extreme case scenarios to better answer national security questions. A more comprehensive validation of the clustered $\mathrm{AOD}_{550}$ data is warranted in the future using experimental AOD data only from 2012 through 2016 and from multiple other data sources (e.g., satellites).
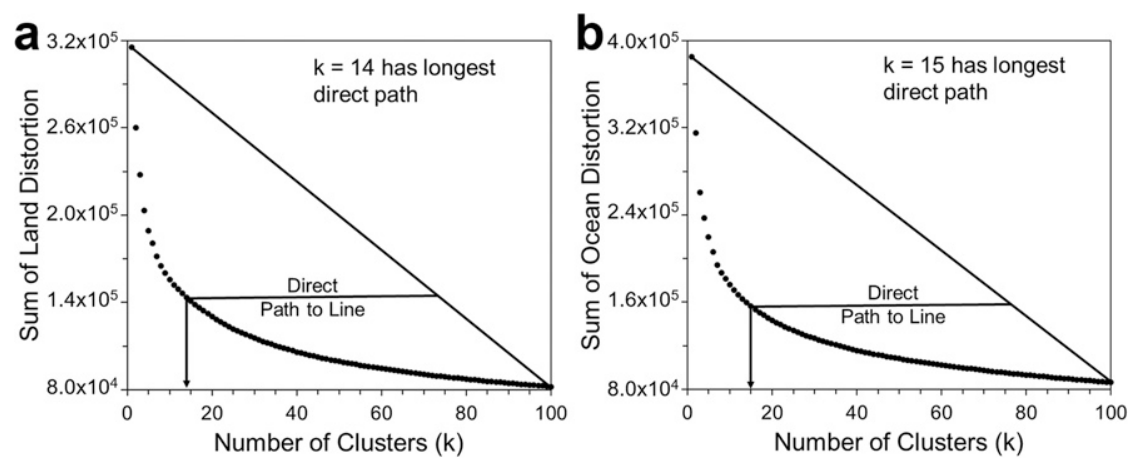

FIG. A1. The sum of the distortion produced by the $k$-means algorithm for all points in all clusters for a given $k$. The $k$-means algorithm was performed on the subset of land and ocean data from the full 2016 MERRA-2 dataset. (a) The distortion for the land dataset; (b) the distortion for the ocean dataset. The point on each plot having the longest direct path to the line connecting the first and last point in the dataset is indicated. 

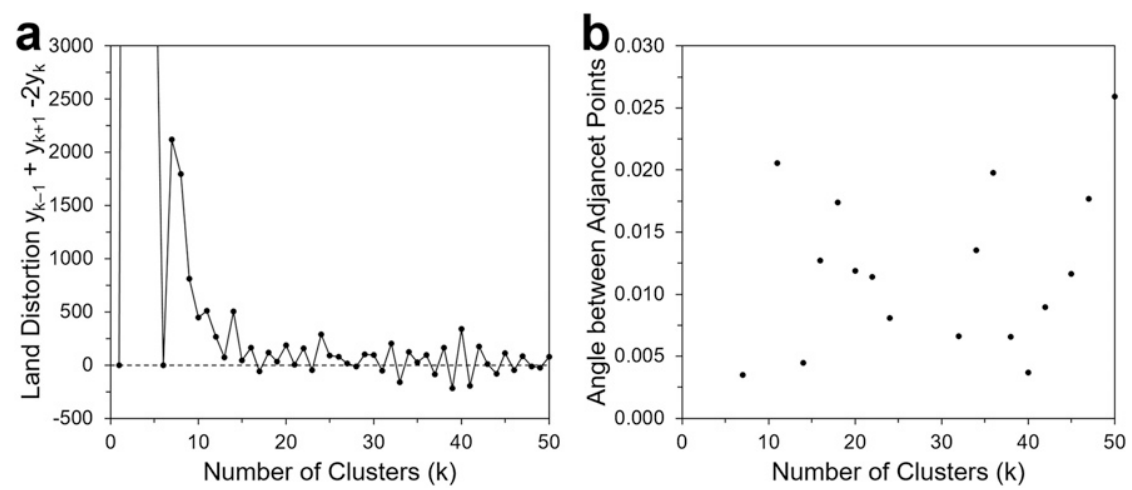

FIG. A2. Geometric angle-based method for the land dataset. (a) Equation (A1) evaluated for the distortion data provided by the $k$-means algorithm performed on the land subset of the 2016 MERRA-2 dataset. Local maxima represent points of maximum curvature in the distortionvs- $k$ plot. (b) The angle (radians) formed by the local maxima in (a) and their two adjacent points.

An additional consideration in interpreting the results is that each of the observation vectors derived from MERRA-2 included in the $k$-means clustering process was weighted equally by the algorithm. However, the natural correlation between some variables could be stronger than for others, and this could be accounted for in principle by applying weights to variables that are known to have greater influence on the resulting AOD. Weights would need to be applied to variables with care to avoid potential bias and incorporate known or deduced relationships between variables. Where such relationships are known a priori, weights could be readily applied to the variables in the clustering process, but an analysis of this nature is outside the scope of this initial work on identifying unique global regions with distinct atmospheric and aerosol conditions.

\section{Conclusions}

The $k$-means clustering algorithm can help identify relationships between variables where no obvious correlation is directly observable, but the algorithm cannot provide an assessment of its performance in retrospect. The results from clustering global variables derived from MERRA-2 reported herein represent approximations of statistical AOD values that are representative of geographic regions with distinct atmospheric properties. The results allow for low (10th percentile), typical (50th percentile), and high (90th percentile) AOD values to be identified easily from lookup tables for quick-turnaround or high-volume applications that can tolerate lower-fidelity AOD information in exchange for simplicity and speed. Moreover, the code books provided for each cluster contain centroid information for all variables included in the clustering algorithm and offer their own insight into the geographic and atmospheric properties of various global regions. In an assessment of the accuracy of the clustered AOD results relative to AERONET data, we determined that there can be a substantial difference between predicted and measured AOD, but the typical error in predicted versus measured AOD mostly falls within the spread of AOD measurements compared. We make clustered data available for the 10th-, 50th-, and 90th-percentile AOD to allow users flexibility in identifying typical and extreme conditions that statistically
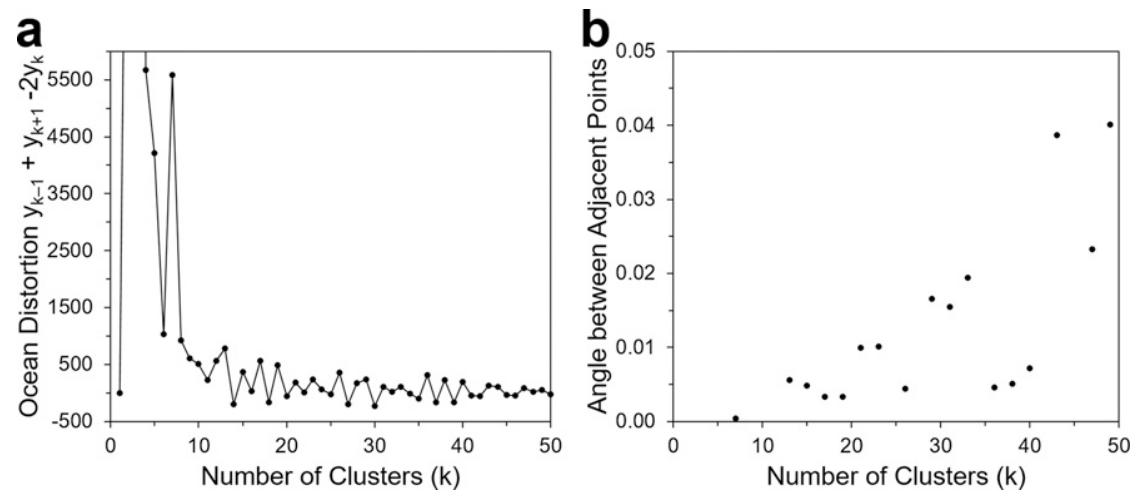

FIG. A3. As in Fig. A2, but for the ocean dataset. 


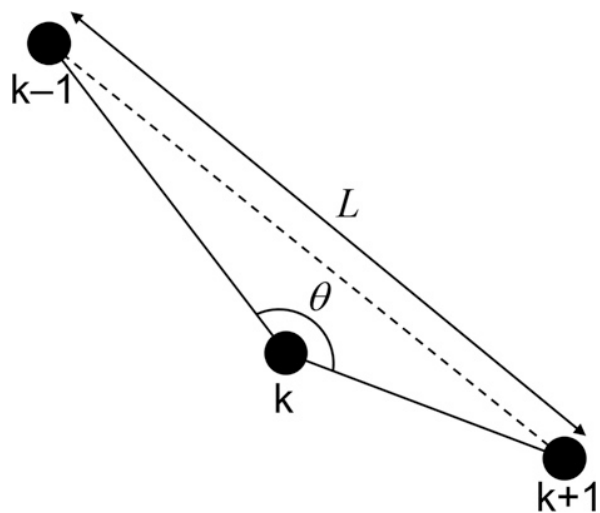

FIG. A4. Depiction of the angle $\theta$ created by three points.

bound the AOD, allowing users of the lookup tables to account for AOD variability as required for their application.

Acknowledgments. This work was supported by the Defense Threat Reduction Agency (DTRA) under contract HQ0034-19-D-0001 and the central research program of the Institute for Defense Analyses. We thank Drs. Joanna Ingraham, Jennifer Bewley, Peter Dolph, Mike Gilmore, Geoffrey Koretsky, Nathan Platt, and Steve Warner for providing helpful feedback over the course of this work.

Data availability statement. Data from MERRA-2 used in this work are available from NASA (https://disc.gsfc.nasa.gov/ datasets) and are described in the MERRA-2 file specification (Bosilovich et al. 2016). Daily average level-2.0 AERONET, version 2, data used in this work are available from AERONET (https://aeronet.gsfc.nasa.gov/; Giles et al. 2019).

\section{APPENDIX A}

\section{Optimizing the Number of Clusters}

The $k$-means clustering algorithm is an unsupervised machine-learning technique that requires the user to input the desired number of clusters, $k$. Organizing large amounts of data into a small number of clusters that represent the dataset as a whole generally involves a trade-off between the computational time to organize the data into clusters and the number of clusters. Ideally, $k$ should be minimized to reduce computation time while maximizing the information contained in the cluster centroids. A way of observing how well the cluster centroids fit the dataset is by calculating the squared distance between each data value in a cluster and its associated centroid, yielding a quantity known as distortion. Distortion decreases and approaches 0 as $k$ approaches the number of data points in the original dataset. In principle, there is an ideal amount of distortion for some value of $k$ that is much smaller than the number of points in the dataset.

Multiple methods have been proposed to optimize $k$ by analyzing the distortion as a function of $k$. These include the "Kneedle" method, a geometric angle-based method, and the Menger curvature method. Additionally, we quantified the Gaussian overlap between the centroids in each cluster as a function of $k$ to identify values for $k$ that yield unique cluster centroids.

\section{a. Optimizing $k$ using the Kneedle method}

It is common practice to optimize $k$ by identifying a "knee" in the distortion-versus- $k$ curve, as shown in Fig. A1 for the 2016 land and ocean MERRA-2 datasets used in this work. In optimizing $k$, we focused on the annual 2016 average MERRA-2 dataset because analysis of multiple years was prohibitive for time and computer resource limitations. The knee or "elbow" of a plot is generally defined as a point of maximum curvature. In some cases, the knee can be identified by visual inspection, but the lack of an obvious knee in this case requires a more quantitative and rigorous analysis.

The Kneedle method (Satopää et al. 2011) for identifying the knee of a curve quantifies the shortest distance between each point in the dataset and a line connecting the two extreme points on the curve and then identifies the point that is farthest away from the line. The result is depicted in Fig. A1. The distortion-versus- $k$ curves presented are averaged for 25 iterations of the $k$-means algo-
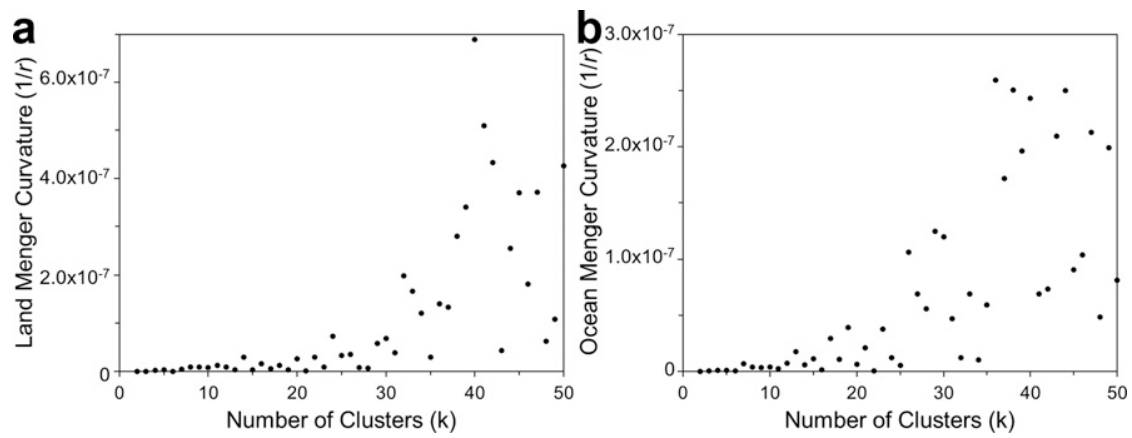

FIG. A5. The Menger curvature is presented for (a) the land distortion-vs- $k$ data in Fig. A1a and (b) the ocean distortion-vs- $k$ data in Fig. A1b as a function of $k$. Points with high Menger curvature have high local curvature in the distortion-vs- $k$ plots. 

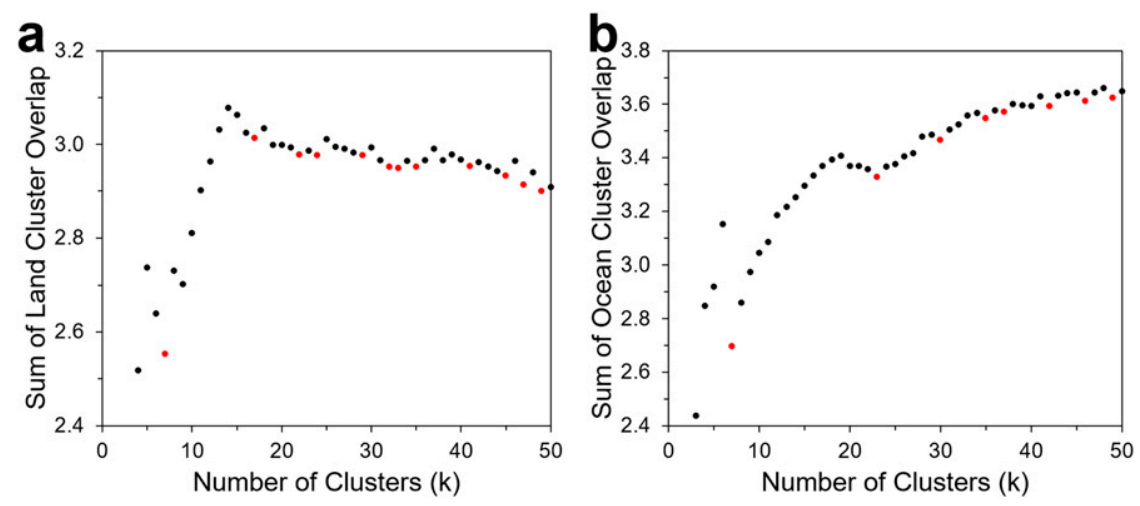

FIG. A6. The sum of the integrated overlap for the Gaussian cluster centroids for (a) the land subset and (b) the ocean subset of the 2016 MERRA-2 dataset as a function of number of clusters. Local minima are indicated in red.

rithm performed on the 2016 dataset. The point on the curve that has the longest distance to the indicated line is $k=14$ for the land dataset and $k=15$ for the ocean dataset. Analysis of the data for fewer iterations of the $k$ means algorithm showed that the optimum $k$ fluctuated between $k=14$ and 15 for both subsets of the MERRA-2 data. Note that the steep negative slope of the line connecting the two extreme points results in the line forming the shortest distance to each data point appearing nearly horizontal.

We found the Kneedle method to be sensitive to the point selected as the maximum number of clusters. For example, if a line is drawn between points at $k=1$ and $k=$ 50 (instead of $k=100$ ), the Kneedle algorithm determines $k=9$ is optimal for both land and ocean datasets. Therefore, we evaluated several other methods to identify local points of maximum curvature in the distortion-versus- $k$ plots.

\section{b. Optimizing $k$ using the geometric angle-based method}

Another method for identifying the point of maximum curvature for a set of data is the geometric angle-based method (Zhao et al. 2008), which compares the $y$ coordinates for point $k$ and the two adjacent points $k-1$ and $k+1$ on the curve. The first step in the geometric anglebased method is to evaluate

$$
y_{k-1}+y_{k+1}-2 y_{k} .
$$

In this application, the $y$ coordinates are the distortion values from the plots given in Fig. A1. The resulting plot of Eq. (A1) evaluated as a function of $k$ is given in Fig. A2a for the 2016 land subset of the MERRA-2 dataset. At local points of maximum curvature, Eq. (A1) evaluates to be greater than the adjacent points, appearing as peaks in Fig. A2a. To determine which peaks represent the most significant curvature in the data as a whole, the angle formed

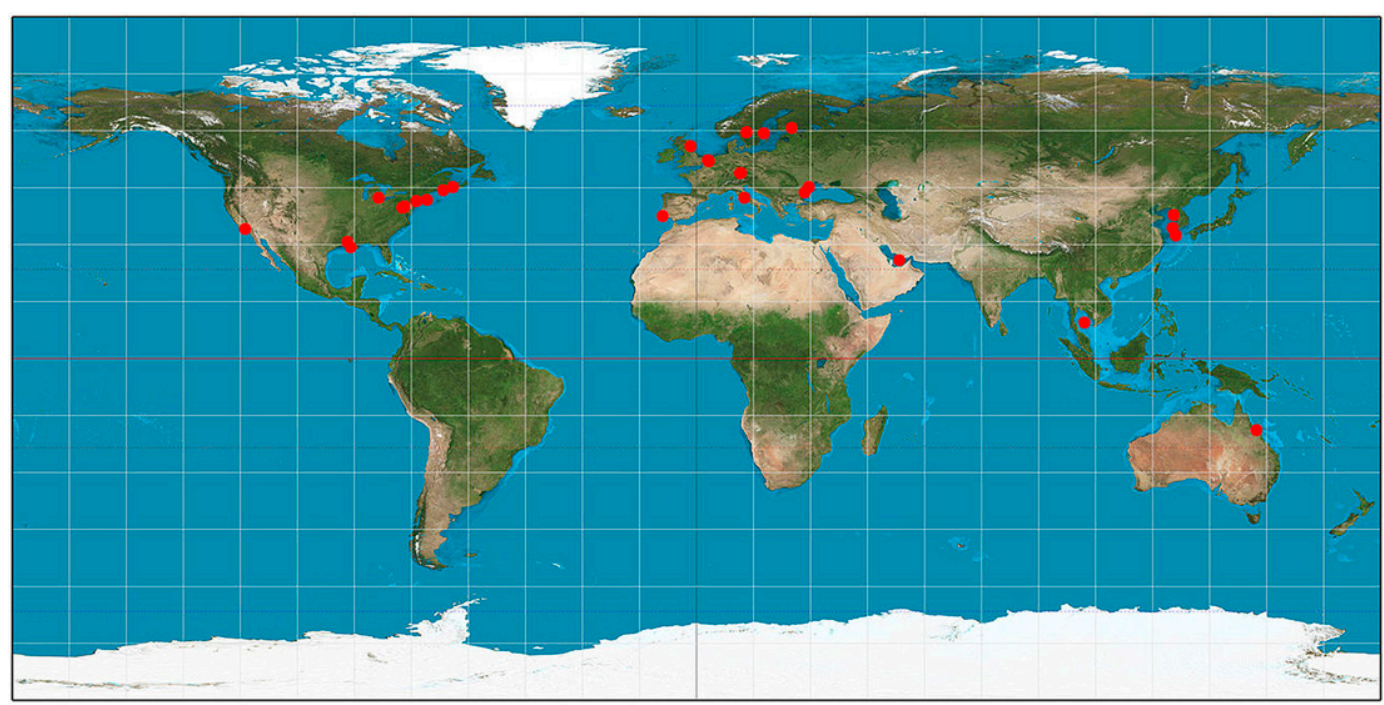

FIG. B1. Locations of the 30 AERONET sites included in the comparison with cluster AOD $_{550}$ values. 

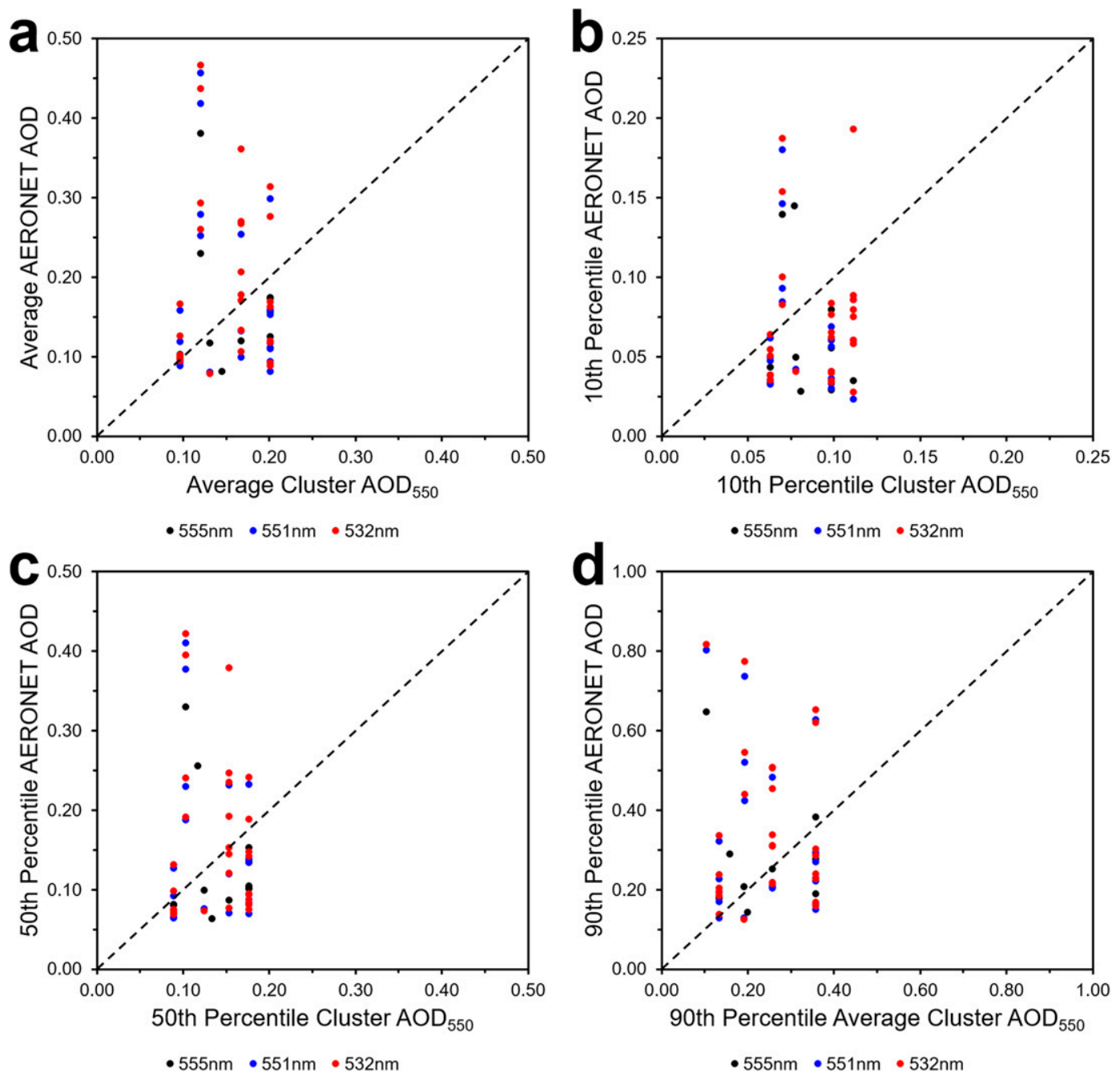

FIG. B2. Annual AERONET AOD statistics vs corresponding 2016 annual cluster AOD $_{550}$ statistics.

between each point in Fig. A2a and its two adjacent points is evaluated and presented in Fig. A2b. The angle data in Fig. A2b is only given where a local maximum is present in Fig. A2a for $k \geq 7$. Where the angle between adjacent points is smallest, the curvature in the distortion dataset is greatest.

Analyzing the local maxima in Fig. A2a in conjunction with the minimum angles found in Fig. A2b indicates that $k=7,14$, and 40 are points where particularly strong curvature is observed in the distortion-versus- $k$ plot for the land subset of the 2016 MERRA-2 data. A broader sample of points with significant curvature include $k=16,20,22,24$, 32 , 38, and 42. This method generally agrees with the Kneedle method for the land dataset, which indicated $k=14$ as a point of maximum curvature.

The results from applying the geometric angle-based method to the ocean subset of the 2016 MERRA-2 dataset are presented in Fig. A3. Similar to the land dataset, points of maximum curvature appear as local maxima in the plot in Fig. A3a. The angle formed by the local maxima and the two adjacent points is given in Fig. A3b for the points having local maximum curvature.

The results presented in Fig. A3 for the ocean dataset indicate that the point exhibiting the highest overall curvature occurs at $k=7$. Other points where local curvature is high occur at $k=13,15,17,19,26,36,38$, and 40. Possibly by coincidence, the results for the ocean dataset agree with the results for the land dataset for where $k=7$ and 40 , but many of the points identified as having high local curvature fall between those identified for the land dataset. Comparing the results of the geometric angle-based method with the results from the Kneedle algorithm performed on the ocean dataset indicates that both methods agree for $k=15$.

\section{c. Optimizing $k$ using the Menger curvature method}

The local curvature for a set of data can be further analyzed using the Menger curvature method (Tolsa 2000). The Menger curvature for three points is the reciprocal of the radius for a circle that passes through the three points. The Menger curvature was evaluated for the distortion-versus- $k$ data from 

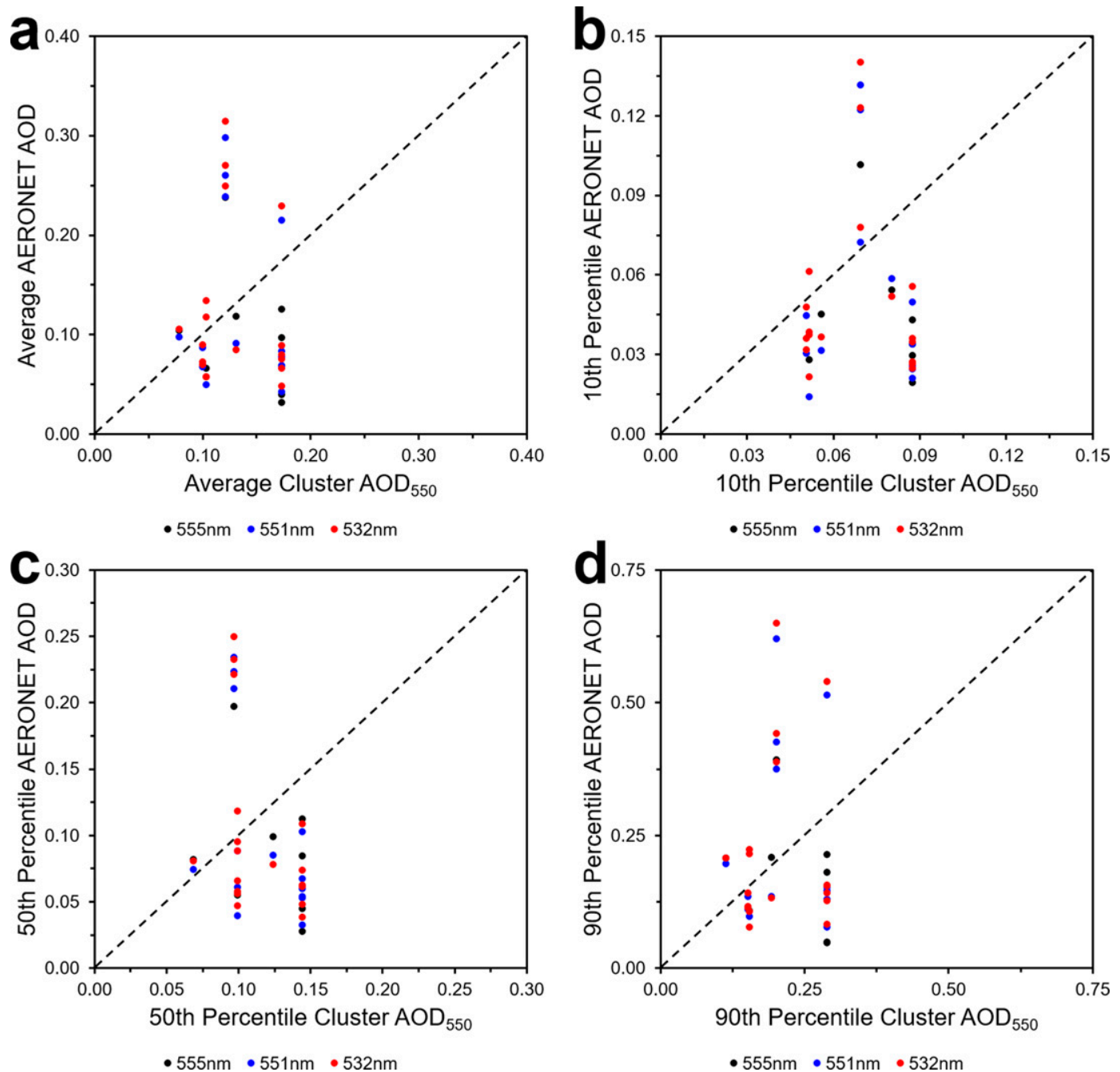

FIG. B3. December-February AERONET AOD statistics vs corresponding 2012-16 December-February cluster AOD 550 statistics.

Fig. A1 to identify local areas of maximum curvature. For each value of $k$, the angle $\theta$ formed by the points at $k-1, k$, and $k+1$ was calculated by evaluating the lengths of the sides of the triangle formed by the three points, as shown in Fig. A4, where the length $L$ is the distance between points at $k-1$ and $k+1$. The radius $r$ of a circle circumscribed by the points that define the triangle in Fig. A4 is given by

$$
r=\frac{L}{2 \sin \theta}
$$

The Menger curvature is calculated by taking the reciprocal of the radius calculated by Eq. (A2). Figure A5 presents the results for the calculation of Menger curvature as a function of $k$ for the land distortion (Fig. A5a) and ocean distortion (Fig. A5b) plots presented in Fig. A1. The steep negative slope of the distortion data results in $\theta$ approaching $180^{\circ}$ ( $\pi$ radians) for many values of $k$, yielding large $r$ and thus small Menger curvature.
The results presented in Fig. A5 indicate values of $k$ for which the local curvature of the distortion-versus- $k$ plots in Fig. A1 is high. The Menger curvature tends to grow significantly greater as $k$ increases, but local maxima in curvature are clearly visible throughout the data. For the land subset, values of $k$ having high Menger curvature include $k=14$, $20,22,24,32$, and 40. These results agree well with the points of maximum curvature determined for the land dataset using the geometric angle-based method presented in Fig. A2. The Menger curvature for the ocean subset shows high curvature at $k=13,17,19,23,26,29,36,38,40$, and 44. Again, there is significant overlap between the results from the Menger curvature method and the geometric angle-based method for finding points of maximum curvature for the ocean dataset.

\section{d. Optimizing $k$ for minimal cluster overlap}

The methods that identify local areas of high curvature in the $k$-means distortion versus $k$ plots do not consider 

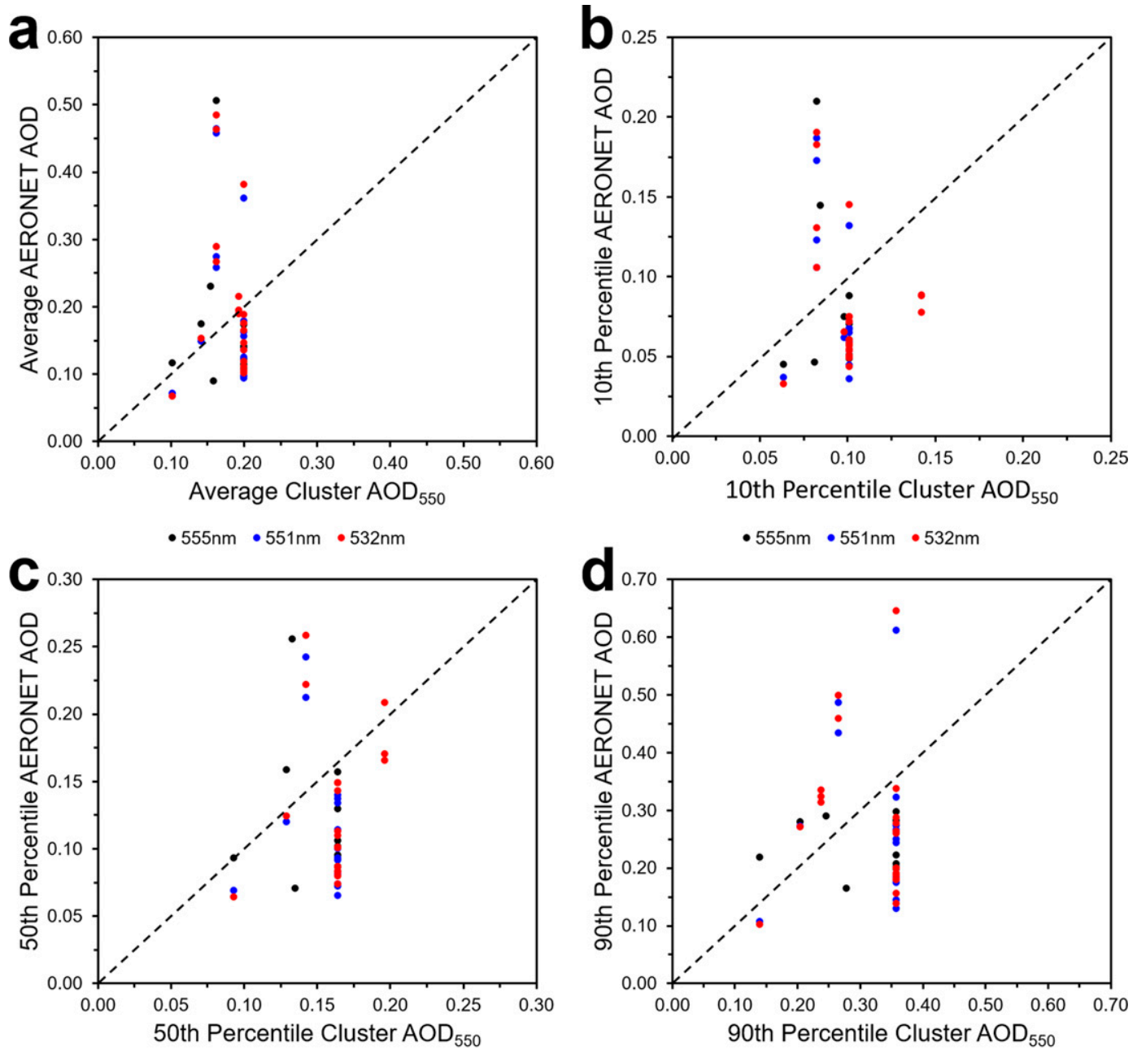

$\bullet 555 \mathrm{~nm} \cdot 551 \mathrm{~nm} \cdot 532 \mathrm{~nm}$

$\bullet 555 \mathrm{~nm} \cdot 551 \mathrm{~nm} \cdot 532 \mathrm{~nm}$

FIG. B4. March-May AERONET AOD statistics vs corresponding 2012-16 March-May cluster AOD 550 statistics.

whether redundant clusters with closely overlapping centroids are produced by the $k$-means algorithm. To identify values of $k$ that produce clusters with minimal overlap, we assumed that the cluster centroids represented the average value for each cluster and that the standard deviation of the data associated with each centroid could be used to represent a Gaussian population. Some centroids were associated with data that had 0 standard deviation. In these cases, we assigned a small standard deviation of $10^{-9}$. The integral overlap between each of the Gaussian cluster centroids was computed for each variable as a function of $k$ and the results are presented in Fig. A6. The results are averaged for 25 iterations of the $k$-means algorithm from $k=1$ to 50 .

The data in Fig. A6 show relatively noisy data, particularly for $k<10$, although the overlap observed for both the land and ocean subsets exhibited a local minimum at $k=$ 7 clusters. For the land subset of the data, the cluster centroid overlap was found to exhibit other local minima at $k=17,22,24,29,32,33,35,41,45,47$, and 49. A slow trend toward lower overlap was observed for the land data for $k>14$, which was the point of maximum overlap. The data from the ocean subset resulted in clusters that had local minimum overlap at $k=23,30,35,37,42,46$, and 49 , and the overlap trended toward a horizontal asymptote as $k$ increased.

\section{e. Comparison of results}

The Kneedle method is convenient because it yields a single answer that it identifies as an optimized value for $k$. However, the observed dependence of this result on the range of the data being analyzed demonstrates that this method has some subjective dependence on user input parameters. Comparison of the results from the Kneedle method with the geometric angle-based method and Menger curvature method allows for a more complete picture of which values of $k$ fall on local "knees" in the distortion versus $k$ curves. These three methods applied to the land subset of the data agree that $k=14$ exhibits high curvature. However, the centroids generated by the $k$-means 

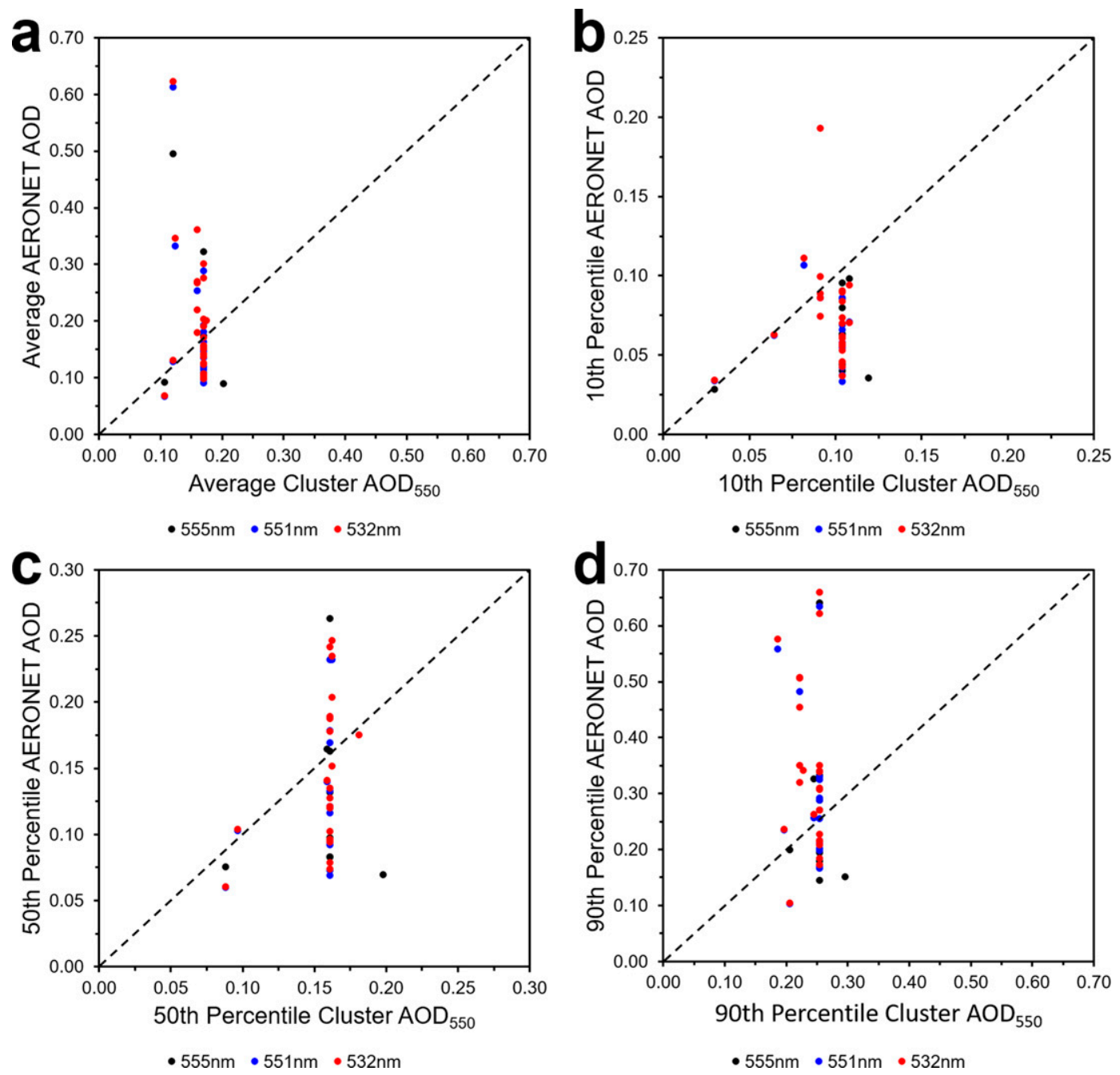

FIG. B5. June-August AERONET AOD statistics vs corresponding 2012-16 June-August cluster AOD 550 statistics.

algorithm for $k=14$ showed the greatest degree of overlap, suggesting that the 14 clusters are least unique.

Of the four techniques used to quantify curvature and cluster overlap, three methods agreed at $k=14,22,24$, and 32 for the land dataset. The methods used to evaluate curvature tended to agree most often, given that they were performed on the same dataset and evaluated the same parameters. The high cluster overlap observed for $k=14$ suggests that this result is not ideal for the purposes of identifying unique and representative clusters. Considering that $k=22,24$, and 32 exhibited similar "knee" behavior and have favorable centroid overlap, we were interested in selecting a number for $k$ that would produce lookup tables containing a manageable amount of meaningful information. Therefore, we selected $k=22$ clusters to represent the land dataset in this work.

The analysis performed to identify optimal values for $k$ for the ocean dataset yielded no consensus where three of the four methods agreed. The ocean data were clustered using the same variables as the land data, but the ocean data consisted of considerably lower variation for the variables that defined the terrain (all points had fraction of ocean $=1$ ) and the elevation. The ocean dataset is, therefore, more uniform and does not require as many clusters as the land dataset. Further, the purpose of defining representative clusters in this work is to allow for the rapid identification of an appropriate $\mathrm{AOD}_{550}$ to use for atmospheric modeling purposes, which we anticipate is of lower interest over the open ocean. The results for optimizing $k$ for the ocean dataset suggest that $k=7$ clusters could be most appropriate. We noted, however, that assigning seven ocean clusters yielded numerous discontinuous regions that were difficult to describe geographically and may add unnecessary bulk to the results. Therefore, we determined that fewer ocean clusters were warranted and settled on using $k=3$ clusters to represent the ocean dataset. 

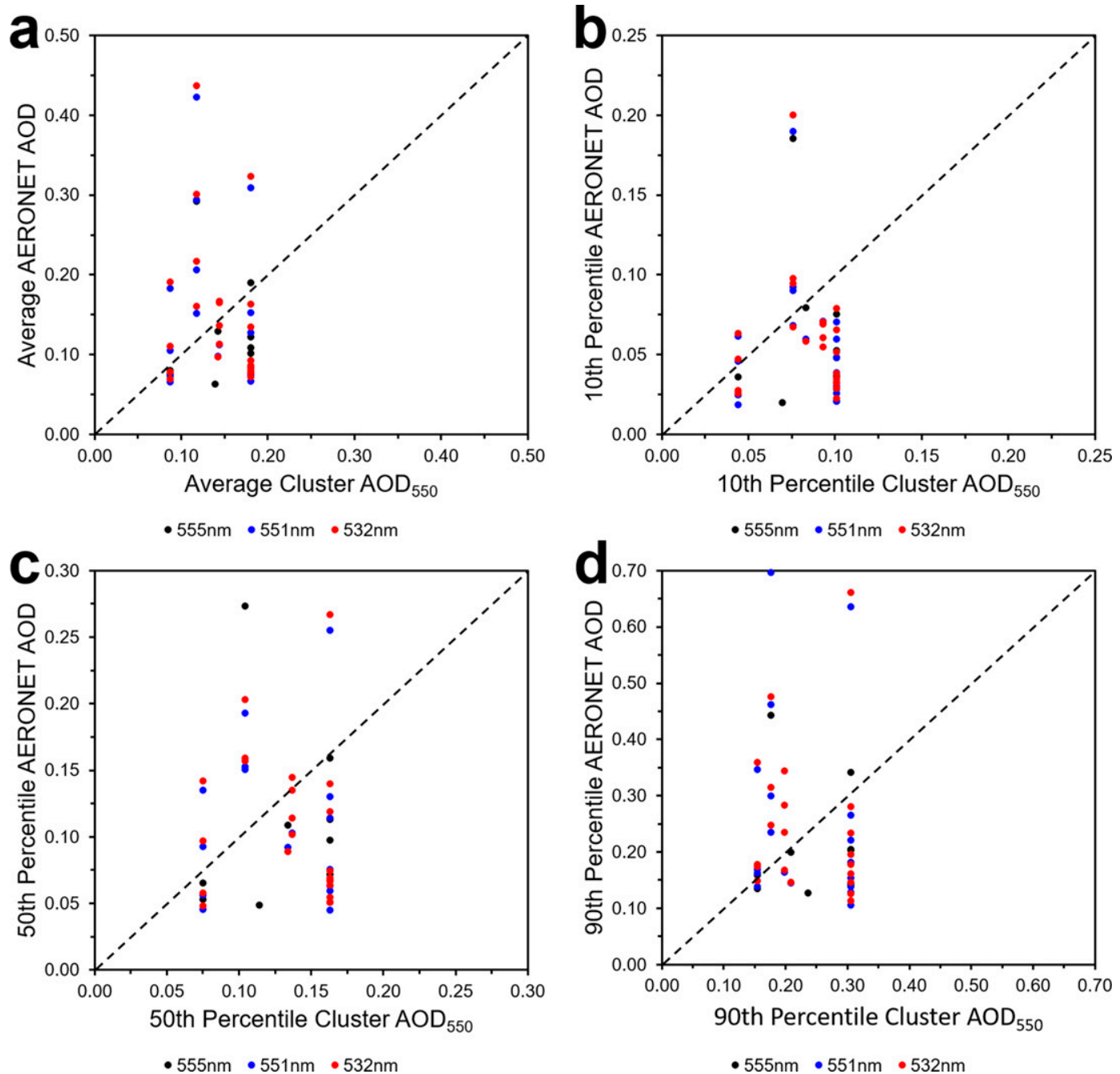

FIG. B6. September-November AERONET AOD statistics vs corresponding 2012-16 September-November cluster AOD 550 statistics.

\section{APPENDIX B}

\section{Comparison of Cluster AOD Predictions with AERONET AOD}

This appendix compares AERONET AOD statistics at 555,551 , and $532 \mathrm{~nm}$ over the respective time of year with the corresponding cluster AOD statistics. The locations of the AERONET sites compared is presented in Fig. B1. We compared the average and the 10th-, 50th-, and 90th-percentile AOD in Figs. B2-B6. The dotted line in each plot represents perfect correlation between experimental AOD and the cluster AOD $_{550}$ derived from MERRA-2. The fractional error given in Table 6 in the main text is represented graphically as the horizontal distance from each point to the dotted line divided by the $y$ axis value of that point (AERONET AOD). We averaged this fractional error for all points in each set of statistics, then averaged the average fractional difference to arrive at the fractional error values in Table 6 .

\section{REFERENCES}

Berk, A., L. S. Bernstein, G. P. Anderson, P. K. Acharya, D. C. Robertson, J. H. Chetwynd, and S. M. Alder-Golden, 1998: MODTRAN cloud and multiple scattering upgrades with application to AVIRIS. Remote Sens. Environ., 65, 367-375, https://doi.org/10.1016/S0034-4257(98)00045-5.

— , G. P. Anderson, P. K. Acharya, and E. P. Shettle, 2011: MODTRAN 5.2.1 User's Manual. Spectral Sciences, 99 pp.

Bosilovich, M. G., R. Lucchesi, and M. Suarez, 2016: MERRA-2: File specification. GMAO Office Note 9 (version 1.1), 73 pp., https://gmao.gsfc.nasa.gov/pubs/docs/Bosilovich785.pdf.

Buchard, V., and Coauthors, 2017: The MERRA-2 aerosol reanalysis, 1980 onward. Part II: Evaluation and case studies. J. Climate, 30, 6851-6872, https://doi.org/10.1175/JCLID-16-0613.1.

Buck, A. L., 1981: New equations for computing vapor pressure and enhancement factor. J. Appl. Meteor., 20, 1527-1532, https://doi. org/10.1175/1520-0450(1981)020<1527:NEFCVP>2.0.CO;2.

Chin, M., and Coauthors, 2002: Tropospheric aerosol optical thickness from the GOCART model and comparisons with 
satellite and sun photometer measurements. J. Atmos. Sci., 59, 461-483, https://doi.org/10.1175/1520-0469(2002)059<0461: TAOTFT $>2.0 . \mathrm{CO} ; 2$.

Colarco, P. R., A. da Silva, M. Chin, and T. Diehl, 2010: Online simulations of global aerosol distributions in the NASA GEOS-4 model and comparisons to satellite and groundbased aerosol optical depth. J. Geophys. Res., 115, D14207, https://doi.org/10.1029/2009JD012820.

Gelaro, R., and Coauthors, 2017: The Modern-Era Retrospective Analysis for Research and Applications, version 2 (MERRA2). J. Climate, 30, 5419-5454, https://doi.org/10.1175/JCLI-D16-0758.1.

Giles, D. M., and Coauthors, 2019: Advancements in the aerosol robotic network (AERONET) version 3 database-Automated near-real-time quality control algorithm with improved cloud screening for sun photometer aerosol optical depth (AOD) measurements. Atmos. Meas. Tech., 12, 169-209, https://doi.org/10.5194/amt-12-169-2019.

Govender, P., and V. Sivakumar, 2019: Application of $k$-means and hierarchical clustering techniques for analysis of air pollution: A review (1980-2019). Atmos. Pollut. Res., 11, 40-56, https://doi.org/10.1016/j.apr.2019.09.009.

Holben, B. N., and Coauthors, 1998: AERONET-A federated instrument network and data archive for aerosol characterization. Remote Sens. Environ., 66, 1-16, https://doi.org/10.1016/ S0034-4257(98)00031-5.

Kinne, S., and Coauthors, 2013: MAC-v1: A new global aerosol climatology for climate studies. J. Adv. Model. Earth Syst., 5, 704-740, https://doi.org/10.1002/jame.20035.

Leroy, S. S., 1997: Measurement of geopotential heights by GPS radio occultation. J. Geophys. Res., 102, 6971-6986, https:// doi.org/10.1029/96JD03083.

Levin, Z., and W. R. Cotton, 2008: Aerosol Pollution Impact on Precipitation: A Scientific Review. Springer Science and Business Media, $386 \mathrm{pp}$.

Liu, Z., and R. George, 2005: Mining weather data using fuzzy cluster analysis. Fuzzy Modeling with Spatial Information for Geographic Problems, F. E. Petry, V. B. Robinson, and M. A. Cobb, Eds., Springer, 105-119, https://doi.org/10.1007/ 3-540-26886-3_5.

Lohmann, U., and J. Feichter, 2005: Global indirect aerosol effects: A review. Atmos. Chem. Phys., 5, 715-737, https://doi. org/10.5194/acp-5-715-2005.

MacQueen, J., 1967: Some methods for classification and analysis of multivariate observations. Statistics, L. M. Le Cam and J. Neyman, Eds., Vol. 1, Proceedings of the Fifth Berkeley Symposium on Mathematical Statistics and Probability, University of California Press, 281-297.

Moosmüller, H., R. K. Chakrabarty, and W. P. Arnott, 2009: Aerosol light absorption and its measurement: A review. $J$. Quant. Spectrosc. Radiat. Transfer, 110, 844-878, https://doi. org/10.1016/j.jqsrt.2009.02.035.

Nabavi, S. O., L. Haimberger, R. Abbasi, and C. Samimi, 2018: Prediction of aerosol optical depth in West Asia using deterministic models and machine learning algorithms. Aeolian Res., 35, 69-84, https://doi.org/10.1016/j.aeolia.2018.10.002.
Prospero, J. M., 1999: Long-term measurements of the transport of African mineral dust to the southeastern United States: Implications for regional air quality. J. Geophys. Res., 104, 15 917-15 927, https://doi.org/10.1029/1999JD900072.

Randles, C. A., and Coauthors, 2017: The MERRA-2 aerosol reanalysis, 1980 onward. Part I: System description and data assimilation evaluation. J. Climate, 30, 6823-6850, https://doi. org/10.1175/JCLI-D-16-0609.1.

Rizza, U., E. Mancinelli, M. Morichetti, G. Passerini, and S. Virgilli, 2019: Aerosol optical depth of the main aerosol species over Italian cities based on the NASA/MERRA-2 model reanalysis. Atmosphere, 10, 709-723, https://doi.org/10.3390/atmos101 10709.

Satheesh, S. K., S. S. Babu, B. Padmakumari, G. Pandithurai, and V. K. Soni, 2017: Variability of atmospheric aerosols over India. Observed Climate Variability and Change over the Indian Region, M. Rajeevan and S. Nayak, Eds., Springer Geology, 221-248, https://doi.org/10.1007/978-981-10-2531-0_13.

Satopää, V., J. Albrecht, D. Irwin, and B. Raghavan, 2011: Finding a "kneedle" in a haystack: Detecting knee points in system behavior. 2011 31st Int. Conf. on Distributed Computing Systems Workshops, Minneapolis, MN, IEEE, 166-171, https://doi.org/10.1109/ICDCSW.2011.20.

Sun, J., and P. R. Ariya, 2006: Atmospheric organic and bio-aerosols as cloud condensation nuclei $(\mathrm{CCN})$ : A review. Atmos. Environ., 40, 795-820, https://doi.org/10.1016/j.atmosenv.2005. 05.052.

Tolsa, X., 2000: Principal values for the Cauchy integral and rectifiability. Proc. Amer. Math. Soc., 128, 2111-2119, https://doi. org/10.1090/S0002-9939-00-05264-3.

Viana, M., J. Pey, X. Querol, A. Alastuey, F. de Leeuw, and A. Lükewille, 2014: Natural sources of atmospheric aerosols influencing air quality across Europe. Sci. Total Environ., 472, 825-833, https://doi.org/10.1016/j.scitotenv.2013.11.140.

Wallace, J. M., and P. V. Hobbs, 2006: Atmospheric thermodynamics. Atmospheric Science, 2nd ed. J. M. Wallace and P. V Hobbs, Eds., Academic Press, 63-111, https://doi.org/10.1016/ B978-0-12-732951-2.50008-9.

Wegner, T., T. Hussein, K. Hämeri, T. Vesala, M. Kulmala, and S. Weber, 2012: Properties of aerosol signature size distributions in the urban environment as derived by cluster analysis. Atmos. Environ., 61, 350-360, https://doi.org/10.1016/j.atmos env.2012.07.048.

Wenny, B. N., J. S. Schafer, J. J. DeLuisi, V. K. Saxena, W. F. Barnard, I. V. Petropavlovskik, and A. J. Vergamini, 1998: A study of regional aerosol radiative properties and effects on ultraviolet-B radiation. J. Geophys. Res., 103, 17083-17097, https://doi.org/10.1029/98JD01481.

Xian, P., and Coauthors, 2019: Current state of the global operational aerosol multi-model ensemble: An update from the International Cooperative for Aerosol Prediction (ICAP). Quart. J. Roy. Meteor. Soc., 145, 176-209, https://doi.org/10. 1002/qj.3497.

Zhao, Q., V. Hautamaki, and P. Fränti, 2008: Knee point detection in BIC for detecting the number of clusters. Advanced Concepts for Intelligent Vision Systems, J. Blanc-Talon et al., Eds., Springer-Verlag, 664-673. 